\title{
Fast GNSS ambiguity resolution as an ill-posed problem
}

Bofeng $\mathrm{Li}^{1}$ Yunzhong Shen ${ }^{1,2}$ Yanming Feng ${ }^{3}$

1: Department of Surveying and Geo-informatics Engineering, Tongji University, Shanghai 200092, China e-mail: bofeng_li@163.com

2: Key Laboratory of Advanced Surveying Engineering of State Bureau of Surveying and Mapping, 1239 Siping Road, 200092 Shanghai, China

e-mail: yzshen@tongji.edu.cn

3: Faculty of Science and Technology, Queensland University of Technology, Brisbane Q4001, Australia e-mail: y.feng@qut.edu.au

Abstract. A linear observational equation system for real time GNSS carrier phase ambiguity resolution (AR) is often severely ill-posed in the case of poor satellite geometry. An ill-posed system may result in unreliable or unsuccessful AR if no care is taken to mitigate this situation. In this paper, the GNSS AR model as an ill-posed problem is solved by regularizing its baseline and ambiguity parameters respectively with the threefold contributions: (i) The regularization parameter is reliably determined in context of minimizing mean square error of regularized solution by replacing the quadratic matrix of the true values of unknowns with the covariance matrix of their initial values; (ii) The different models for computing initial values of unknowns are systematically discussed in order to address the potential schemes in real world applications; (iii) The superior performance of the regularized AR are demonstrated through the numerically random simulations as well as the real GPS experiments. The results show that the proposed regularization strategies can effectively mitigate the model's ill-condition and improve the success AR probability of the observational system with a severely ill-posed problem.

Keywords. GNSS; ambiguity resolution; regularization; ill-posed problem; success probability

\section{Introduction}

GNSS carrier phase integer ambiguity resolution (AR) as a key to precise real time positioning applications has attracted a great deal of research attentions since early 1980s. Many methods and algorithms have been developed, including extra-widelaning technique (Wübbena 1989), ambiguity function method (Counselman and Gourevitch 1981), fast ambiguity resolution approach (Frei and Beutler 1990), Cholesky decomposition (Euler and Landau 1992; Xu 2001), least squares ambiguity searching technique (Hatch 1990), Least squares AMBiguity Decorrelation Adjustment (LAMBDA) (Teunissen 1993) as well as Ambiguity Resolution with Constraint Equations (ARCE) method (Park et al. 1996), of which the LAMBDA is popularly used in the geodetic community due to its efficient search speed (Teunissen 1999). A good AR method is characterized by the following aspects (Abidin 1993; Chen 1994; Xu 1998a): 
(i) Computed float ambiguities are close to their integers and their covariance matrix is near diagonal. In other words, the error correlation between the float ambiguities is generally weak. To achieve this, any available constraint between coordinates or ambiguities may be made use of, depending on the situation whether a prior baseline information is given or a subset of ambiguity integers are predetermined (Li and Shen 2009). Alternatively, many researchers take the ill-condition of the GNSS AR model into account, using the regularization against least squares (LS) estimation to obtain more accurate float ambiguity solutions (Xu et al. 1999; Ou and Wang 2004; Shen and Li 2007; Gui and Han 2007; Li and Shen 2008).

(ii) As the core of an AR process, the integer search strategy can efficiently determine the integers. A good search engine can, to a certain extent, reduce the quality demand on the float solutions. In fact, this has been the focus of the most of AR methods, of which the integer rounding is the simplest way to immediately fix the ambiguities to their nearest integers, but it cannot achieve high success probability since the correlations amongst all ambiguities are not considered. Also using rounding but partially considering correlation between two consecutive ambiguities, Dong and Bock (1989) proposed a bootstrapping procedure based on the sequential LS adjustment. In the bootstrapping, a number of the nuisance ambiguity candidates can be excluded and the search efficiency is improved. Teunissen (1993) firstly attempted to rigorously estimate ambiguities using integer LS (ILS) method which considers the correlations amongst all ambiguities in the integer search process. Furthermore, Teunissen (1995) systematically established the LAMBDA method with an embedded decorrelation technique to improve the integer search speed. Afterwards, several alternative decorrelation techniques have been proposed, such as LLL, inverse integer Cholesky decomposition as well as united ambiguity decorrelation (Xu et al. 1995; Liu et al. 1999; Grafarend 2000; Xu 2001; Chang et al. 2005). However, it is realized by Xu (2001) that all decorrelation techniques can speed up the estimation procedure only if the dimension of the integer vector is not too high. Therefore, how to efficiently improve the search speed in high dimensional case is still an open and challenging issue.

(iii) The reliability of integer solutions from the searching engine is evaluated with a robust hypothesis testing. This is an important and also rather challenging issue. It is important because a wrong integer solution can lead to incorrect estimation of the remaining real parameters, while difficult because the integer is discrete and one cannot describe its reliability using uncertainty like a real parameter. Many research efforts have been made to address this issue based on the AR success probability, including early contributions by Hassibi and Boyd (1996, 1998), Teunissen (1998, 1999, 2001, 2002), Verhagen (2004, 2005) and more recent works by Xu (2006), O’Keefe et al. (2007), Zhu et al. (2007).

A linear observational system for fast or real time GNSS AR and position estimation is often severely ill-posed due to the poor observation geometry in short observation span. As a result, a small observational error may result in a 
large error in the LS solution, where the float ambiguities distinctly apart from their integers and the covariance matrix is characterized by strong correlation between ambiguity parameters. In fact, the stable float solution is possible when the model's ill-condition is effectively mitigated. In the past few years, several researchers have attempted to solve the problem for stable float ambiguities by regularizing this ill-posed model (Xu et al. 1999; Ou and Wang 2004; Shen and Li 2007; Gui and Han 2007; Li and Shen 2008). Ou and Wang (2004) studied a regularized AR with a regularization matrix constructed from the baseline part of the normal equations. The estimation requires a prior knowledge of the baseline vector at the decimeter-level and the regularization parameter (RP) is empirically chosen to 1 . Shen and Li (2007) proposed to regularize the ambiguity parameters to stabilize their float estimates as well. Gui and Han (2007) put forward a double- $k$ type regularization method to regularize baseline and ambiguities simultaneously using two different RPs. In fact, the double- $k$ type regularization is essentially equivalent to the single- $k$ type regularization, because once one type of the parameters is reliably estimated, the remaining can be computed accordingly. With a prior knowledge of baseline, a more reasonable RP can be determined (Li and Shen 2008).

However, there have been problems in using regularization to solve the GNSS AR model in the past works. (i) RP is crucial for the regularization but rather difficult to determine because in theory the true values of unknowns are needed in the minimizing the mean square error (MSE) criterion, whereas no sufficient attentions have been paid to this issue in the existing works. (ii) Regularization can indeed make the float solution decorrelated and its probability density function (PDF) sharper, but the regularized solution is biased. Nevertheless in the previous works, the regularized bias is ignored. The question is that whether the regularized biases are considerable to affect the efficiency of regularized AR.

In this paper, a fast GNSS AR model as an ill-posed problem is solved by regularizing its baseline and ambiguity parameters respectively to address the problems identified thus far. To determine a reliable PR, we replace the quadratic matrix of the true values of unknowns with the covariance matrix of their initial values, and thus the problem of determining the RP turns to a problem of computing the stable initial values of unknowns. Furthermore, the different models for computing initial values of unknowns are systematically discussed in order to address the potential schemes in real applications. Since it is rather difficult to explicitly prove the higher success probability of regularized AR if considering the regularized biases, the random simulations as well as the real GPS experiments are adopted to numerically demonstrate superior performance of regularized AR.

The rest of the paper is organized as follows. Section 2 briefly introduces the regularization estimation for ill-posed model. This is followed by the theoretical analysis on the basis for using the covariance matrix of the initial values of unknowns instead of the quadratic matrix of true values in the RP determination. Section 3 gives the fast GNSS AR 
model and demonstrates the ill-posed characteristics of its LS solution. In sections 4 and 5, we will give more comprehensive investigation into the regularized AR by regularizing the baseline and ambiguity parameters respectively, and the potential schemes in real applications are discussed. The random simulations and the real GPS experiments are implemented in sections 6 and 7 to demonstrate the superior performance of regularized AR. The concluding remarks are outlined in the last section.

\section{Ill-posed problem and regularization estimation}

\subsection{Mathematical model and regularization estimation}

A linear (linearized) model is expressed as

$$
\boldsymbol{y}=\boldsymbol{A x}+\boldsymbol{e} ; \quad \sigma_{0}^{2} \boldsymbol{Q}
$$

where $\boldsymbol{y} \in \mathbb{R}^{n}$ is an observation vector contaminated by an error vector $\boldsymbol{e} \in \mathbb{R}^{n}$ with normal distribution of mean zero and covariance matrix $\sigma_{0}^{2} \boldsymbol{Q} ; \sigma_{0}^{2}$ is a variance scalar of unit weight and $\boldsymbol{W}=\boldsymbol{Q}^{-1}$ a positive-definite weight matrix; $\boldsymbol{A} \in \mathbb{R}^{n \times m}$ is a matrix with full column rank connected to the unknown vector $\boldsymbol{x} \in \mathbb{R}^{m}$ and generally $n>m$. If the matrix $\boldsymbol{A}$ is well-conditioned, the LS solution as the best unbiased estimation to this overdetermined system of Eq.(1) is given as

$$
\widehat{\boldsymbol{x}}_{L}=\left(\boldsymbol{A}^{T} \boldsymbol{W} \boldsymbol{A}\right)^{-1} \boldsymbol{A}^{T} \boldsymbol{W} \boldsymbol{y}
$$

However, if $\boldsymbol{A}$ is ill-posed (namely the condition number of $\boldsymbol{A}$ is very large), the LS solution (2) becomes instable because a small error in the observation vector $\boldsymbol{y}$ will derive a large error in the solution $\widehat{\boldsymbol{x}}_{L}$. In order to stabilize the solution in such ill-posed model, Tikhonov (1963) regularization is commonly used, which is also known as ridge regression in statistics. The regularization solves the problem (1) with the following minimization

$$
\min _{\boldsymbol{x}}\|\boldsymbol{A x}-\boldsymbol{y}\|_{\boldsymbol{Q}}^{2}+\alpha\|\boldsymbol{x}\|^{2}
$$

where $\|\cdot\|_{\boldsymbol{Q}}^{2}=(\cdot)^{T} \boldsymbol{Q}^{-1}(\cdot)$ and $\alpha>0$ is the RP. The regularized solution is derived as

$$
\widehat{\boldsymbol{x}}_{R}=\left(\boldsymbol{A}^{T} \boldsymbol{W} \boldsymbol{A}+\alpha \boldsymbol{I}_{m}\right)^{-1} \boldsymbol{A}^{T} \boldsymbol{W} \boldsymbol{y}=\boldsymbol{N}_{\alpha}^{-1} \boldsymbol{A}^{T} \boldsymbol{W} \boldsymbol{y} ; \quad \boldsymbol{\Sigma}_{\widehat{\boldsymbol{x}}_{R}}=\sigma_{0}^{2} \boldsymbol{N}_{\alpha}^{-1} \boldsymbol{N}_{\boldsymbol{x}} \boldsymbol{N}_{\alpha}^{-1}
$$

where $\boldsymbol{N}_{\boldsymbol{x}}=\boldsymbol{A}^{T} \boldsymbol{W} \boldsymbol{A}$ and $\boldsymbol{N}_{\alpha}=\boldsymbol{A}^{T} \boldsymbol{W} \boldsymbol{A}+\alpha \boldsymbol{I}_{m}$ with $\boldsymbol{I}_{m}$ being the $m \times m$ identity matrix. The regularized solution is biased and its bias is computed as

$$
\boldsymbol{g}_{\widehat{\boldsymbol{x}}_{R}}=E\left(\widehat{\boldsymbol{x}}_{R}-\boldsymbol{x}\right)=-\alpha \boldsymbol{N}_{\alpha}^{-1} \overline{\boldsymbol{x}}
$$

(see e.g., Shen and $\mathrm{Li} 2007$ ), where $E(\cdot)$ is the expectation operation and $\overline{\boldsymbol{x}}$ is the true value of the unknown vector. Traditionally, MSE is used to evaluate the regularized solution including the effect of the bias term

$$
\boldsymbol{M}_{\widehat{\boldsymbol{x}}_{R}}=E\left[\left(\widehat{\boldsymbol{x}}_{R}-\boldsymbol{x}\right)\left(\widehat{\boldsymbol{x}}_{R}-\boldsymbol{x}\right)^{T}\right]=\boldsymbol{\Sigma}_{\widehat{\boldsymbol{x}}_{R}}+\boldsymbol{g}_{\widehat{\boldsymbol{x}}_{R}} \boldsymbol{g}_{\widehat{\boldsymbol{x}}_{R}}^{T}=\boldsymbol{N}_{\alpha}^{-1}\left(\sigma_{0}^{2} \boldsymbol{N}_{x}+\alpha^{2} \overline{\boldsymbol{x} \boldsymbol{x}^{T}}\right) \boldsymbol{N}_{\alpha}^{-1}
$$

(see e.g., Xu and Rummel 1994). Desirably, a good estimation is companied by a small bias besides a stable solution. Since the true values $\overline{\boldsymbol{x}}$ is never known, as suggested by Xu et al. (2006) it can be directly replaced by the regularized 
estimate to compute the regularized bias as

$$
\widehat{\boldsymbol{g}_{\widehat{\boldsymbol{x}}_{R}}}=-\alpha \boldsymbol{N}_{\alpha}^{-1} \widehat{\boldsymbol{x}}_{R}
$$

Since $\widehat{\boldsymbol{x}}_{R}$ is a biased estimate of $\boldsymbol{x}$, we have

$$
\boldsymbol{g}_{\widehat{\boldsymbol{x}}_{\widehat{x}_{R}}}=E\left(\widehat{\boldsymbol{g}}_{\widehat{\boldsymbol{x}}_{R}}-\boldsymbol{g}_{\widehat{\boldsymbol{x}}_{R}}\right)=\alpha^{2} \boldsymbol{N}_{\alpha}^{-2} \overline{\boldsymbol{x}}
$$

Obviously, if we use $\widehat{\boldsymbol{x}}_{R}$ to estimate the biases of $\widehat{\boldsymbol{x}}_{R}$, the estimation error is the second-order values of RP Thus it is reasonable to evaluate the regularized bias using Eq. (8) if RP is small.

\subsection{Determination of regularization parameter}

The RP $\alpha$ is prerequisite for the regularized solution. There are many methods for computing RP, such as, discrepancy principle, general cross validation, L-curve and so on (Hansen 1992; Xu 1998b). In this paper, we compute a RP by minimizing MSE of the regularized solution to guarantee the estimation efficiency, namely,

$$
\alpha=\arg \min _{\alpha>0} \operatorname{tr}\left(\boldsymbol{M}_{\widehat{\boldsymbol{x}}_{R}}\right)
$$

where $\operatorname{tr}(\cdot)$ is the mathematical operation for computing the trace of a matrix. It is easy to prove that the second-order derivative $\partial^{2} \operatorname{tr}\left(\boldsymbol{M}_{\widehat{\boldsymbol{x}}_{R}}\right) / \partial \alpha^{2}>0$ holds true for any $\alpha>0$, which means that the unique minimization point exists. It is observed that the true values $\overline{\boldsymbol{x}}$ are necessary to compute a RP, but in practice they are never known. One can replace $\overline{\boldsymbol{x}}$ by their initial values (for instance, the LS estimates), namely, $\overline{\boldsymbol{x}}^{T} \approx \widehat{\boldsymbol{x}} \widehat{\boldsymbol{x}}^{T}$ with $\widehat{\boldsymbol{x}}$ being the initial values. It is crucial to determine $\operatorname{RP} \alpha$, because the regularized solution is sensitive to $\alpha$. From theoretical point of view, $\alpha$ is used to essentially balance the contributions of observation and regularized bias to the regularized solution. A large $\alpha$ value will definitely lead to a large bias. Conversely, if $\alpha$ value is too small, the model's ill-condition cannot be effectively mitigated and then the regularized solution is still unstable. Apparently, if we use the non-iterative initial solution to compute $\alpha$, the initial values are usually large such that the derived $\alpha$ is too small. Thus the initial solution should be computed iteratively. However, after iteration, the systematic trends are basically removed from observations, i.e., $E(\widehat{\boldsymbol{x}}) \approx \mathbf{0}$. Hence we have the approximation

$$
\widehat{\boldsymbol{x}} \widehat{\boldsymbol{x}}^{T}=(\widehat{\boldsymbol{x}}-E(\widehat{\boldsymbol{x}}))(\widehat{\boldsymbol{x}}-E(\widehat{\boldsymbol{x}}))^{T} \approx E\left[(\widehat{\boldsymbol{x}}-E(\widehat{\boldsymbol{x}}))(\widehat{\boldsymbol{x}}-E(\widehat{\boldsymbol{x}}))^{T}\right]=\sigma_{0}^{2} \boldsymbol{Q}_{\widehat{\boldsymbol{x}}}
$$

and the RP can be in practice computed by

$$
\alpha=\arg \min _{\alpha>0} \operatorname{tr}\left(\boldsymbol{N}_{\alpha}^{-1}\left(\boldsymbol{N}_{\boldsymbol{x}}+\alpha^{2} \boldsymbol{Q}_{\widehat{\boldsymbol{x}}}\right) \boldsymbol{N}_{\alpha}^{-1}\right)
$$

One may use the LS solution $\widehat{\boldsymbol{x}}_{L}$ to replace $\overline{\boldsymbol{x}}$, namely, LS covariance matrix $\sigma_{0}^{2} \boldsymbol{Q}_{\widehat{\boldsymbol{x}}_{L}}$ to replace $\overline{\boldsymbol{x}}^{T}$. However, in ill-posed model (1), the cofactor matrix $\boldsymbol{Q}_{\widehat{x}_{L}}$ is generally unstable. Therefore, alternative methods to compute the stable initial values of unknowns and their cofactor matrix are adopted in the context of GNSS AR in next section. As a consequence, the determination of RP is turned to seek the stable initial estimates and their cofactor matrix. 


\section{Fast GPS ambiguity resolution model and integer least squares estimation}

The double differenced (DD) GPS observation model for a short baseline and its LS estimation are given. Then the ill-posed characteristics of ambiguity normal equations for a short observation span are demonstrated.

\subsection{GPS observation model for a short baseline}

We start from the linearized DD phase equations at a short baseline ignoring the effects of residual atmospheric biases

$$
\boldsymbol{\Phi}_{j}=\left[\begin{array}{ll}
\boldsymbol{A}_{j} & \boldsymbol{B}_{j}
\end{array}\right]\left[\begin{array}{l}
\boldsymbol{a} \\
\boldsymbol{b}
\end{array}\right]+\boldsymbol{e}_{\boldsymbol{\Phi}_{j}} ; \quad \sigma_{0}^{2} \boldsymbol{Q}_{\boldsymbol{\Phi}_{j}}
$$

where $\boldsymbol{\Phi}_{j}$ and $\boldsymbol{e}_{\boldsymbol{\Phi}_{j}}$ are the vectors of phase observations and their noises; $\boldsymbol{a} \in \mathbb{Z}^{m}$ and $\boldsymbol{b} \in \mathbb{R}^{3}$ are the unknown vectors for integer ambiguities and baseline respectively, and their corresponding coefficient matrices are $\boldsymbol{A}_{j}=\lambda \boldsymbol{I}_{m}$ and $\boldsymbol{B}_{j} ; \sigma_{0}^{2}$ is the variance of DD phase serving as a prior variance of unit weight; $\boldsymbol{Q}_{\boldsymbol{\Phi}_{j}}$ is a cofactor matrix of phase observations. The subscript “” denotes the $j$ th epoch. Similarly, the DD pseudorange equations read

$$
\boldsymbol{P}_{j}=\boldsymbol{B}_{j} \boldsymbol{b}+\boldsymbol{e}_{\boldsymbol{P}_{j}} ; \quad \sigma_{0}^{2} \boldsymbol{Q}_{\boldsymbol{P}_{j}}
$$

where the cofactor matrix of pseudoranges $\boldsymbol{Q}_{\boldsymbol{P}_{j}}=\kappa \times \boldsymbol{Q}_{\boldsymbol{\Phi}_{j}}$ and $\kappa=\sigma_{C}^{2} / \sigma_{0}^{2}$ is a scalar weight for pseudorange relative to phase; $\sigma_{C}^{2}$ is the variance of DD pseudorange; Collecting $n$ epoch observations together yields

$$
\boldsymbol{y}=\boldsymbol{A} \boldsymbol{a}+\boldsymbol{B} \boldsymbol{b}+\boldsymbol{e} ; \quad \sigma_{0}^{2} \boldsymbol{Q}
$$

where $\boldsymbol{y}=\left[\begin{array}{llllll}\boldsymbol{\Phi}_{1}^{T} & \cdots & \boldsymbol{\Phi}_{n}^{T} & \boldsymbol{P}_{1}^{T} & \cdots & \boldsymbol{P}_{n}^{T}\end{array}\right]^{T}, \boldsymbol{e}=\left[\begin{array}{llllll}\boldsymbol{e}_{\boldsymbol{\Phi}_{1}}^{T} & \cdots & \boldsymbol{e}_{\boldsymbol{\Phi}_{n}}^{T} & \boldsymbol{e}_{\boldsymbol{P}_{1}}^{T} & \cdots & \boldsymbol{e}_{\boldsymbol{P}_{n}}^{T}\end{array}\right]^{T}, \boldsymbol{A}=\left[\begin{array}{l}1 \\ 0\end{array}\right] \otimes\left[\begin{array}{c}\boldsymbol{A}_{1} \\ \vdots \\ \boldsymbol{A}_{n}\end{array}\right], \boldsymbol{B}=\left[\begin{array}{l}1 \\ 1\end{array}\right] \otimes$ $\left[\begin{array}{c}\boldsymbol{B}_{1} \\ \vdots \\ \boldsymbol{B}_{n}\end{array}\right] ; \boldsymbol{Q}=\left[\begin{array}{cc}\boldsymbol{I}_{n} & \mathbf{0} \\ \mathbf{0} & \kappa \times \boldsymbol{I}_{n}\end{array}\right] \otimes \boldsymbol{Q}_{\boldsymbol{\Phi}}$ is a cofactor matrix with $\boldsymbol{Q}_{\boldsymbol{\Phi}}=\boldsymbol{Q}_{\boldsymbol{\Phi}_{1}}=\cdots=\boldsymbol{Q}_{\boldsymbol{\Phi}_{n}} ; \boldsymbol{A}_{1}=\cdots=\boldsymbol{A}_{n}=\lambda \boldsymbol{I}_{m}$ and $\lambda$ is the wavelength of phase. The weight matrix $\boldsymbol{W}=\boldsymbol{Q}^{-1}$.

\subsection{Integer least squares ambiguity resolution}

Being different from purely real-valued model, Eq.(14) includes integer ambiguity parameters. The ILS method was introduced by Teunissen (1993) to solve Eq. (14). An alternative two-step procedure was described in Xu et al. (1995). In this paper, we outline the formulae of the float solution and integer search criterion directly without any derivation. The normal equations of LS float solutions are

$$
\left[\begin{array}{ll}
\boldsymbol{A}^{T} \boldsymbol{W} \boldsymbol{A} & \boldsymbol{A}^{T} \boldsymbol{W} \boldsymbol{B} \\
\boldsymbol{B}^{T} \boldsymbol{W} \boldsymbol{A} & \boldsymbol{B}^{T} \boldsymbol{W} \boldsymbol{B}
\end{array}\right]\left[\begin{array}{l}
\widehat{\boldsymbol{a}}_{L} \\
\widehat{\boldsymbol{b}}_{L}
\end{array}\right]=\left[\begin{array}{l}
\boldsymbol{A}^{T} \boldsymbol{W} \boldsymbol{y} \\
\boldsymbol{B}^{T} \boldsymbol{W} \boldsymbol{y}
\end{array}\right]
$$

For simplifying the expressions, we set up the notations: $\left[\begin{array}{ll}\boldsymbol{N}_{\boldsymbol{a} a \boldsymbol{a}} & \boldsymbol{N}_{\boldsymbol{a b}} \\ \boldsymbol{N}_{\boldsymbol{b} \boldsymbol{a}} & \boldsymbol{N}_{\boldsymbol{b} \boldsymbol{b}}\end{array}\right]=\left[\begin{array}{ll}\boldsymbol{A}^{T} \boldsymbol{W} \boldsymbol{A} & \boldsymbol{A}^{T} \boldsymbol{W} \boldsymbol{B} \\ \boldsymbol{B}^{T} \boldsymbol{W} \boldsymbol{A} & \boldsymbol{B}^{T} \boldsymbol{W} \boldsymbol{B}\end{array}\right],\left[\begin{array}{l}\boldsymbol{u}_{\boldsymbol{a}} \\ \boldsymbol{u}_{\boldsymbol{b}}\end{array}\right]=\left[\begin{array}{l}\boldsymbol{A}^{T} \boldsymbol{W} \boldsymbol{y} \\ \boldsymbol{B}^{T} \boldsymbol{W} \boldsymbol{y}\end{array}\right]$; $N_{a \mid b}=N_{a a}-N_{a b} N_{b b}^{-1} N_{b a}, \quad N_{b \mid a}=N_{b b}-N_{b a} N_{a a}^{-1} N_{a b} ; u_{b \mid a}=u_{b}-N_{b a} N_{a a}^{-1} u_{a}, u_{a \mid b}=u_{a}-N_{a b} N_{b b}^{-1} u_{b}$ and $\left[\begin{array}{cc}\boldsymbol{Q}_{\widehat{\boldsymbol{a}}_{L}} & \boldsymbol{Q}_{\widehat{\boldsymbol{a}}_{L} \widehat{\boldsymbol{b}}_{L}} \\ \boldsymbol{Q}_{\widehat{\boldsymbol{b}}_{L}, \widehat{\boldsymbol{a}}_{L}} & \boldsymbol{Q}_{\widehat{\boldsymbol{b}}_{L}}\end{array}\right]=\left[\begin{array}{cc}\boldsymbol{N}_{\boldsymbol{a} a} & \boldsymbol{N}_{\boldsymbol{a} \boldsymbol{b}} \\ \boldsymbol{N}_{\boldsymbol{b a}} & \boldsymbol{N}_{\boldsymbol{b} \boldsymbol{b}}\end{array}\right]^{-1}$. Here the subscript “L” denotes the LS solution. Thus the LS float solution is 
symbolized from Eq.(15) as

$$
\widehat{\boldsymbol{a}}_{L}=\boldsymbol{N}_{\boldsymbol{a} \mid \boldsymbol{b}}^{-1} \boldsymbol{u}_{\boldsymbol{a} \mid \boldsymbol{b}}, \boldsymbol{\Sigma}_{\widehat{\boldsymbol{a}}_{L}}=\sigma_{0}^{2} \boldsymbol{N}_{\boldsymbol{a} \mid \boldsymbol{b}}^{-1}
$$

In the second step, the float ambiguities are mapped to their integers by solving the minimization problem (Teunissen 1993; Xu et al. 1995)

$$
\breve{\boldsymbol{a}}_{L}=\arg \min _{\boldsymbol{a} \in \mathbb{Z}^{m}}\left(\widehat{\boldsymbol{a}}_{L}-\boldsymbol{a}\right)^{T} \boldsymbol{\Sigma}_{\widehat{\boldsymbol{a}}_{L}}^{-1}\left(\widehat{\boldsymbol{a}}_{L}-\boldsymbol{a}\right)
$$

Because the integer is discrete, we cannot drive an explicit expression of solution unless the covariance matrix $\boldsymbol{\Sigma}_{\widehat{a}_{L}}$ is diagonal. Thus the searching procedure is applied to pick out the optimal integer candidate. To speed up the search, the decorrelation technique is often employed to make the strongly correlated covariance matrix $\boldsymbol{\Sigma}_{\widehat{a}_{L}}$ towards a diagonal one (Teunissen 1993, 1995; Xu et al. 1995; Liu et al. 1999; Grafarend 2000; Xu 2001; Chang et al. 2005). From the geometric view, it makes the elongated super-ellipsoid more sphere-like.

\subsection{Ill-posed characteristic of ambiguity normal equations}

In fast GNSS AR, the normal equations (15) are strongly collinear due to the strong correlation between baseline and ambiguity parameters (Teunissen and Kleusberg 1998; Li and Shen 2010). In other words, if three baseline components or at least three DD ambiguities are fixed, the remaining parameters can be computed accordingly. From the spectrum point of view, there are three extremely small eigenvalues within all eigenvalues of the norm matrix. We apply singular value decomposition (SVD) for the normal matrix associated to ambiguities as

$$
\boldsymbol{N}_{\boldsymbol{a} \mid \boldsymbol{b}}=\boldsymbol{V} \boldsymbol{\Theta} \boldsymbol{V}^{T}
$$

where $\boldsymbol{\Theta}=\operatorname{diag}\left(\theta_{1} \quad \cdots \quad \theta_{m}\right)$ satisfies with $\theta_{1}>\cdots>\theta_{m}$ and last three components $\theta_{m-2}, \theta_{m-1}$ and $\theta_{m}$ are much smaller than the others. To intuitively illustrate this ill-posed property, we collect 1000 epochs of L1 phase data from a $4.6 \mathrm{~km}$ baseline (which is also used in the latter experiments in section 7). The 7 common satellites (i.e., 6 ambiguities) are tracked in the whole computations. The ambiguity normal equations are computed using 10 epoch data and the oldest epoch is updated for each computation. All eigenvalues for total 991 experiments are shown in Figure 1 where the y-axis denotes the common algorithm of eigenvalues. Each subplot shows all 991 computations for one eigenvalue. Apparently, the variation in each subplot is very small and smooth due to the smooth satellite geometry variation, but the differences between the first three and the last three eigenvalues are significant, far beyond a scale of $10^{-4}$. Therefore, the normal matrix $\boldsymbol{N}_{\boldsymbol{a} \mid \boldsymbol{b}}$ in fast GNSS AR is severely ill-posed with property that three eigenvalues of $N_{a \mid b}$ are extremely smaller than the others. 


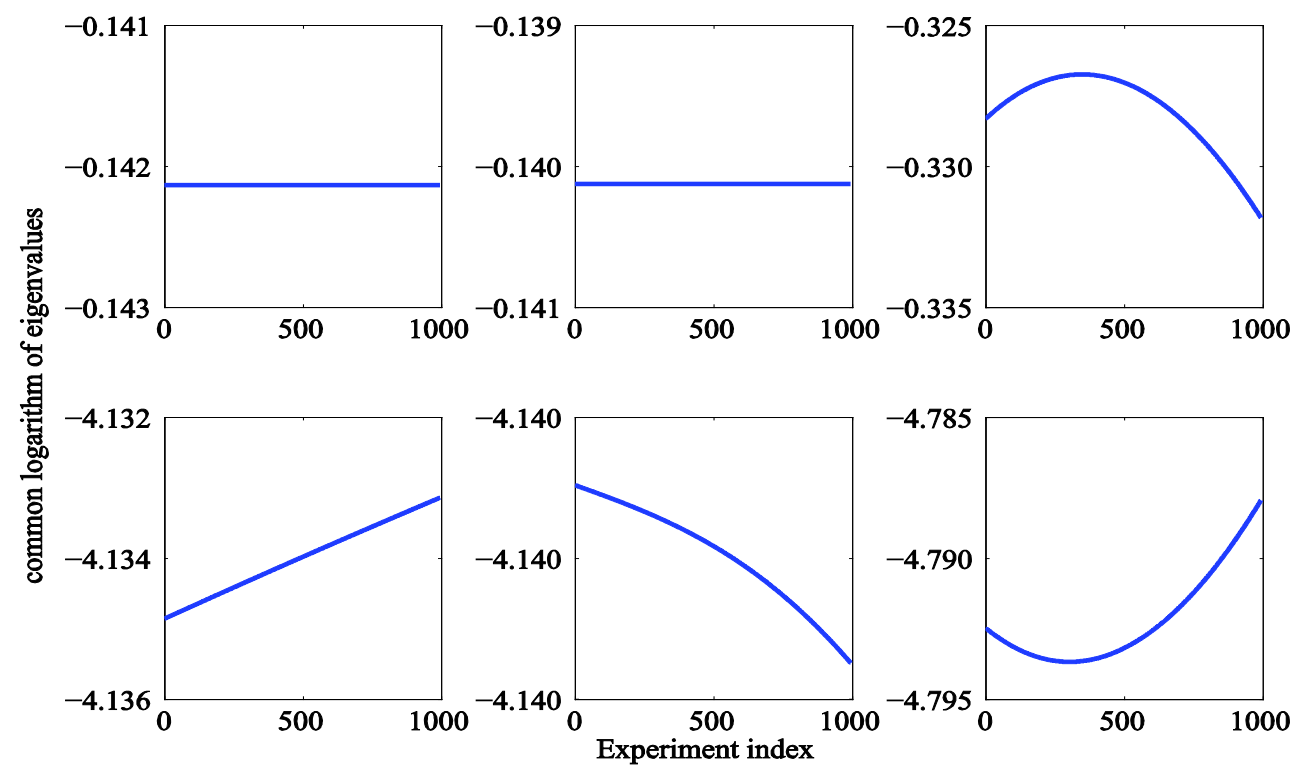

Figure 1: Eigenvalue variations of the ambiguity normal matrices over 1000 data epochs

\section{Ambiguity estimation by regularizing baseline parameters}

Following from the above analysis on the ill-posed fast GNSS AR problem, we introduce the regularization algorithm to this ill-posed model, namely regularizing the baseline parameters.

\subsection{Mathematical model}

The minimization function contains an additional quadratic term of the baseline vector

$$
\min _{\boldsymbol{a}, \boldsymbol{b}}\|\boldsymbol{A} \boldsymbol{a}+\boldsymbol{B} \boldsymbol{b}-\boldsymbol{y}\|_{\boldsymbol{Q}}^{2}+\alpha\|\boldsymbol{b}\|^{2}, \boldsymbol{a} \in \mathbb{R}^{\boldsymbol{m}}
$$

The regularized baseline solutions are

$$
\widehat{\boldsymbol{b}}_{R B}=\widetilde{\boldsymbol{N}}_{\boldsymbol{b} \mid \boldsymbol{a}}^{-1} \boldsymbol{u}_{\boldsymbol{b} \mid \boldsymbol{a}} ; \quad \boldsymbol{\Sigma}_{\widehat{\boldsymbol{b}}_{R B}}=\sigma_{0}^{2} \widetilde{\boldsymbol{N}}_{\boldsymbol{b} \mid \boldsymbol{a}}^{-1} \boldsymbol{N}_{\boldsymbol{b} \mid \boldsymbol{a}} \widetilde{\boldsymbol{N}}_{\boldsymbol{b} \mid \boldsymbol{a}}^{-1}
$$

where $\widetilde{\boldsymbol{N}}_{\boldsymbol{b} \mid \boldsymbol{a}}=\boldsymbol{N}_{\boldsymbol{b} \mid \boldsymbol{a}}+\alpha \boldsymbol{I}_{3}$ and the subscript " $R B$ " denotes the regularized solution by regularizing baseline parameters. Accordingly, the regularized bias is computed as

$$
\boldsymbol{g}_{\widehat{\boldsymbol{b}}_{R B}}=E\left(\widehat{\boldsymbol{b}}_{R B}-\boldsymbol{b}\right)=-\alpha \widetilde{\boldsymbol{N}}_{\boldsymbol{b} \mid \boldsymbol{a}}^{-1} \overline{\boldsymbol{b}}
$$

It is easy to derive the regularized float ambiguities by substituting Eq.(20) into Eq.(15),

$$
\widehat{\boldsymbol{a}}_{R B}=\boldsymbol{N}_{\boldsymbol{a} \boldsymbol{a}}^{-1}\left(\boldsymbol{u}_{\boldsymbol{a}}-\boldsymbol{N}_{\boldsymbol{a b}} \widehat{\boldsymbol{b}}_{R B}\right) ; \boldsymbol{\Sigma}_{\widehat{\boldsymbol{a}}_{R B}}=\sigma_{0}^{2}\left(\boldsymbol{N}_{\boldsymbol{a} \boldsymbol{a}}-\boldsymbol{N}_{\boldsymbol{a b}} \widetilde{\boldsymbol{N}}_{\boldsymbol{b} \boldsymbol{b}}^{-1} \boldsymbol{N}_{\boldsymbol{b a}}\right)^{-1}
$$

with $\widetilde{\boldsymbol{N}}_{\boldsymbol{b} \boldsymbol{b}}=\boldsymbol{N}_{\boldsymbol{b} \boldsymbol{b}}+\alpha \boldsymbol{I}_{3}$. Similarly, the biases of regularized ambiguities are

$$
\boldsymbol{g}_{\widehat{\boldsymbol{a}}_{R B}}=\boldsymbol{N}_{\boldsymbol{a} \boldsymbol{a}}^{-1} \boldsymbol{N}_{\boldsymbol{a} \boldsymbol{b}} \boldsymbol{g}_{\widehat{b}_{R B}}=\alpha \boldsymbol{N}_{\boldsymbol{a} \boldsymbol{a}}^{-1} \boldsymbol{N}_{\boldsymbol{a} \boldsymbol{b}} \widetilde{\boldsymbol{N}}_{\boldsymbol{b} \mid \boldsymbol{a}}^{-1} \bar{b}
$$

and their MSE are

$$
\boldsymbol{M}_{\widehat{\boldsymbol{a}}_{R B}}=\sigma_{0}^{2}\left(\boldsymbol{N}_{\boldsymbol{a} a}-\boldsymbol{N}_{\boldsymbol{a b}} \widetilde{\boldsymbol{N}}_{\boldsymbol{b} \boldsymbol{b}}^{-1} \boldsymbol{N}_{\boldsymbol{b a}}\right)^{-1}+\alpha^{2} \boldsymbol{N}_{\boldsymbol{a} a \boldsymbol{a}}^{-1} \boldsymbol{N}_{\boldsymbol{a b}} \widetilde{\boldsymbol{N}}_{\boldsymbol{b} \mid \boldsymbol{a}}^{-1} \overline{\boldsymbol{b}} \overline{\boldsymbol{b}}^{T} \widetilde{\boldsymbol{N}}_{\boldsymbol{b} \mid \boldsymbol{a}}^{-1} \boldsymbol{N}_{\boldsymbol{b a}} \boldsymbol{N}_{\boldsymbol{a} \boldsymbol{a}}^{-1}
$$

Following the RP computation practice with Eq.(11), $\overline{\boldsymbol{b}} \overline{\boldsymbol{b}}^{T}$ is substituted by $\sigma_{0}^{2} \boldsymbol{Q}_{\widehat{\boldsymbol{b}}}$ to derive the RP as 


$$
\alpha=\arg \min _{\alpha>0} \operatorname{tr}\left[\left(\boldsymbol{N}_{\boldsymbol{a} a}-\boldsymbol{N}_{\boldsymbol{a b}} \widetilde{\boldsymbol{N}}_{\boldsymbol{b} \boldsymbol{b}}^{-1} \boldsymbol{N}_{\boldsymbol{b a}}\right)^{-1}+\alpha^{2} \boldsymbol{N}_{\boldsymbol{a} a}^{-1} \boldsymbol{N}_{\boldsymbol{a} \boldsymbol{b}} \widetilde{\boldsymbol{N}}_{\boldsymbol{b} \mid \boldsymbol{a}}^{-1} \boldsymbol{Q}_{\widetilde{\boldsymbol{b}}} \widetilde{\boldsymbol{N}}_{\boldsymbol{b} \mid \boldsymbol{a}}^{-1} \boldsymbol{N}_{\boldsymbol{b a}} \boldsymbol{N}_{a \boldsymbol{a}}^{-1}\right]
$$

Thereby, the key to computing a RP turns to computation of a stable initial baseline and its covariance matrix.

\subsection{Initial baseline computed from pseudorange observables}

To obtain a stable initial baseline, we make use of pseudoranges in the geometry-based and time-averaged models, respectively. The geometry-based model is referred to as an observation model involving geometric parameters such as coordinates etc.; whereas a time-averaged model is a simplified geometry-based model where the several consecutive geometry-based equations are averaged due to a small geometry variation in a short time span. Comparing with geometry-based model, the compatible solution is obtained in time-averaged model, but the computation efficiency can be significantly improved (Teunissen 1997).

Geometry-based model

The initial baseline is computed using the pseudoranges of multiple epochs based on geometry-based model as

$$
\widehat{\boldsymbol{b}}_{G B}=\left(\sum_{j=1}^{n} \boldsymbol{B}_{j}^{T} \boldsymbol{Q}_{\boldsymbol{P}_{j}}^{-1} \boldsymbol{B}_{j}\right)^{-1}\left(\sum_{j=1}^{n} \boldsymbol{B}_{j}^{T} \boldsymbol{Q}_{\boldsymbol{P}_{j}}^{-1} \boldsymbol{P}_{j}\right)
$$

and its corresponding covariance matrix is derived as

$$
\boldsymbol{\Sigma}_{\widehat{\boldsymbol{b}}_{G B}}=\sigma_{0}^{2} \kappa\left(\sum_{j=1}^{n} \boldsymbol{B}_{j}^{T} \boldsymbol{Q}_{\boldsymbol{\Phi}_{j}}^{-1} \boldsymbol{B}_{j}\right)^{-1}
$$

where the subscript “ ${ }_{G B}$ ” indicates geometry-based model.

\section{Time-averaged model}

The time-averaged model is used to efficiently compute baseline as

$$
\widehat{\boldsymbol{b}}_{T A}=\left(\overline{\boldsymbol{B}}^{T} \boldsymbol{Q}_{\boldsymbol{P}}^{-1} \overline{\boldsymbol{B}}\right)^{-1}\left(\overline{\boldsymbol{B}}^{T} \boldsymbol{Q}_{\boldsymbol{P}}^{-1} \overline{\boldsymbol{P}}\right)
$$

where $\overline{\boldsymbol{B}}=\frac{1}{n} \sum_{j=1}^{n} \boldsymbol{B}_{j}$ and $\overline{\boldsymbol{P}}=\frac{1}{n} \sum_{j=1}^{n} \boldsymbol{P}_{j}$. The subscript “ТА” denotes the time-averaged model. Accordingly, its covariance matrix is derived as

$$
\boldsymbol{\Sigma}_{\widehat{\boldsymbol{b}}_{T A}}=\frac{\sigma_{0}^{2} \kappa}{n}\left(\overline{\boldsymbol{B}}^{T} \boldsymbol{Q}_{\boldsymbol{\Phi}}^{-1} \overline{\boldsymbol{B}}\right)^{-1}
$$

\section{Ambiguity estimation by regularizing ambiguity parameters}

As alternative scheme, we can stabilize the fast GNSS AR model by regularizing the ambiguity parameters. In this scheme, not only the geometry-based and time-averaged but also the geometry-free models are employed to compute the initial ambiguities for RP determination.

\subsection{Mathematical model}

The regularized AR based on regularizing ambiguities is solved by minimizing the following cost function

$$
\min _{\boldsymbol{a}, \boldsymbol{b}}\|\boldsymbol{A} \boldsymbol{a}+\boldsymbol{B} \boldsymbol{b}-\boldsymbol{y}\|_{\boldsymbol{Q}}^{2}+\alpha\|\boldsymbol{a}\|^{2}, \boldsymbol{a} \in \mathbb{R}^{m}
$$


The regularized float ambiguities read

$$
\widehat{\boldsymbol{a}}_{R A}=\widetilde{\boldsymbol{N}}_{\boldsymbol{a} \mid \boldsymbol{b}}^{-1} \boldsymbol{u}_{\boldsymbol{a} \mid \boldsymbol{b}} ; \quad \boldsymbol{\Sigma}_{\widehat{\boldsymbol{a}}_{R A}}=\sigma_{0}^{2} \widetilde{\boldsymbol{N}}_{\boldsymbol{a} \mid \boldsymbol{b}}^{-1} \boldsymbol{N}_{\boldsymbol{a} \mid \boldsymbol{b}} \widetilde{\boldsymbol{N}}_{\boldsymbol{a} \mid \boldsymbol{b}}^{-1}
$$

where $\widetilde{\boldsymbol{N}}_{\boldsymbol{a} \mid \boldsymbol{b}}=\boldsymbol{N}_{\boldsymbol{a} \mid \boldsymbol{b}}+\alpha \boldsymbol{I}_{m}$ and the subscript “RA” denotes the regularized solution by regularizing ambiguity parameters. The regularized bias is computed in terms of Eq.(5),

$$
\boldsymbol{g}_{\widehat{\boldsymbol{a}}_{R A}}=E\left(\widehat{\boldsymbol{a}}_{R A}-\boldsymbol{a}\right)=-\alpha \widetilde{\boldsymbol{N}}_{\boldsymbol{a} \mid \boldsymbol{b}}^{-1} \overline{\boldsymbol{a}}
$$

The MSE is computed according to Eq.(6) by

$$
\boldsymbol{M}_{\widehat{\boldsymbol{a}}_{R A}}=\sigma_{0}^{2} \widetilde{\boldsymbol{N}}_{\boldsymbol{a} \mid \boldsymbol{b}}^{-1} \boldsymbol{N}_{\boldsymbol{a} \mid \boldsymbol{b}} \widetilde{\boldsymbol{N}}_{\boldsymbol{a} \mid \boldsymbol{b}}^{-1}+\alpha^{2} \widetilde{\boldsymbol{N}}_{\boldsymbol{a} \mid \boldsymbol{b}}^{-1} \overline{\boldsymbol{a}} \overline{\boldsymbol{a}}^{T} \widetilde{\boldsymbol{N}}_{\boldsymbol{a} \mid \boldsymbol{b}}^{-1}
$$

Following the similar RP computation to Eq. (25), $\overline{\boldsymbol{a}} \overline{\boldsymbol{a}}^{T}$ is replaced by the covariance matrix $\boldsymbol{\Sigma}_{\widehat{\boldsymbol{a}}}$ of initial ambiguities, we have the RP for ambiguity vector as follows

$$
\alpha=\operatorname{argmin}_{\alpha>0} \operatorname{tr}\left[\widetilde{\boldsymbol{N}}_{\boldsymbol{a} \mid \boldsymbol{b}}^{-1}\left(\boldsymbol{N}_{\boldsymbol{a} \mid \boldsymbol{b}}+\alpha^{2} \boldsymbol{Q}_{\widehat{\boldsymbol{a}}}\right) \widetilde{\boldsymbol{N}}_{\boldsymbol{a} \mid \boldsymbol{b}}^{-1}\right]
$$

\subsection{Initial ambiguities computed from pseudoranges}

We compute the initial ambiguities by fixing the initial baseline derived from the geometry-based and time-averaged models respectively. In addition, the geometry-free model is used to compute the initial ambiguities. As defined by Teunissen (1997), the geometry-free model is the simplest model for ambiguity estimation where the geometry-specific parameters are all canceled.

Geometry-based model

The ambiguities are computed using the baseline solved from the geometry-based model

$$
\widehat{\boldsymbol{a}}_{G B}=\left(\overline{\boldsymbol{\Phi}}-\overline{\boldsymbol{B}} \widehat{\boldsymbol{b}}_{G B}\right) / \lambda
$$

with $\overline{\boldsymbol{\Phi}}=\frac{1}{n} \sum_{j=1}^{n} \boldsymbol{\Phi}_{j}$, and their corresponding covariance matrix is

$$
\boldsymbol{\Sigma}_{\widehat{\boldsymbol{a}}_{G B}}=\frac{\sigma_{0}^{2}}{\lambda^{2}}\left[\frac{Q_{\boldsymbol{\Phi}}}{n}+\kappa \overline{\boldsymbol{B}}\left(\sum_{j=1}^{n} \boldsymbol{B}_{j}^{T} \boldsymbol{Q}_{\boldsymbol{\Phi}_{j}}^{-1} \boldsymbol{B}_{j}\right)^{-1} \overline{\boldsymbol{B}}^{T}\right]
$$

Time-averaged model

If the time-averaged baseline $\widehat{\boldsymbol{b}}_{T A}$ is used instead of $\widehat{\boldsymbol{b}}_{G B}$ in Eq.(35), the covariance matrix of initial ambiguities becomes

$$
\boldsymbol{\Sigma}_{\widehat{\boldsymbol{a}}_{T A}}=\frac{\sigma_{0}^{2}}{\lambda^{2} \times n}\left[\boldsymbol{Q}_{\boldsymbol{\Phi}}+\kappa \overline{\boldsymbol{B}}\left(\overline{\boldsymbol{B}}^{T} \boldsymbol{Q}_{\boldsymbol{\Phi}}^{-1} \overline{\boldsymbol{B}}\right)^{-1} \overline{\boldsymbol{B}}^{T}\right]
$$

Geometry-free model

If only phase is used in error equations, we can alternatively compute initial ambiguities using geometry-free model where geometric parameters are canceled. Thus the initial ambiguities are computed as

$$
\widehat{\boldsymbol{a}}_{G F}=(\overline{\boldsymbol{\Phi}}-\overline{\boldsymbol{P}}) / \lambda
$$

and their covariance matrix is derived as 


$$
\boldsymbol{\Sigma}_{\widehat{\boldsymbol{a}}_{G F}}=\frac{(1+\kappa) \sigma_{0}^{2}}{\lambda^{2} \times n} \boldsymbol{Q}_{\boldsymbol{\Phi}}
$$

\section{Evaluation of regularized ambiguity resolution}

The superior performance of regularized AR would be evident from its success probability. However the regularized solution is biased and it is very difficult to explicitly prove its higher success probability relative to that of LS. Hence we illustrate this benefit of the regularized integer solutions by random simulations in the following discussions.

\subsection{Shannon's upper probabilistic bound for ambiguity resolution}

The essence of fixing ambiguity is to map a real-valued float solution onto its integer value. Due to the discrete property of integer ambiguity, multiple float ambiguities could be mapped onto a unique integer. Hassibi and Boyd (1996, 1998) introduced the Voronoi cell to describe the set of the float ambiguities that corresponds to a unique integer, see also Xu (2006), which is alternatively called pull-in region by Teunissen (1999, 2001) in GNSS community. If the float ambiguity is within this Voronoi cell, it can be correctly fixed into its integer, otherwise mapped onto another integer. In other words, the integral probability of the float ambiguity over the Voronoi cell is equivalent to the success probability of correct AR. Obviously, the PDF of the float ambiguities and the Voronoi cell must be given to compute the success probability.

Considering the normally distributed error vector $\boldsymbol{e}$, the float solution $\widehat{\boldsymbol{a}}_{L}$ is of normal distribution, i.e., $\widehat{\boldsymbol{a}}_{L} \sim N\left(\begin{array}{ll}\overline{\boldsymbol{a}} & \boldsymbol{\Sigma}_{\widehat{\boldsymbol{a}}_{L}}\end{array}\right)$ with $\overline{\boldsymbol{a}}$ and $\boldsymbol{\Sigma}_{\widehat{\boldsymbol{a}}_{L}}$ the expectation and covariance matrix of $\widehat{\boldsymbol{a}}_{L}$, respectively. Thus the probability of LS AR is

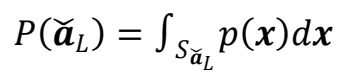

where the PDF of LS float solution $p(\boldsymbol{x})=\left|2 \pi \boldsymbol{\Sigma}_{\widehat{\boldsymbol{a}}_{L}}\right|^{-1 / 2} \exp \left\{-\frac{1}{2}(\boldsymbol{x}-\overline{\boldsymbol{a}})^{T} \boldsymbol{\Sigma}_{\widehat{\boldsymbol{a}}_{L}}^{-1}(\boldsymbol{x}-\overline{\boldsymbol{a}})\right\}$ and $S_{\widetilde{\boldsymbol{a}}_{L}}$ is the Voronoi cell of integer solution $\breve{\boldsymbol{a}}_{L} \cdot|\cdot|$ represents the determinant operation of a matrix. If $\breve{\boldsymbol{a}}_{L}$ is assumed to be the unknown true integer solution, then the integral (40) becomes the success probability (Xu 2006)

$$
P\left(\check{\boldsymbol{a}}_{L}\right)=\int_{S_{0, L}} p(\boldsymbol{x}) d \boldsymbol{x}
$$

and $p(\boldsymbol{x})=\left|2 \pi \boldsymbol{\Sigma}_{\widehat{\boldsymbol{a}}_{L}}\right|^{-1 / 2} \exp \left\{-\frac{1}{2} \boldsymbol{x}^{T} \boldsymbol{\Sigma}_{\widehat{\boldsymbol{a}}_{L}}^{-1} \boldsymbol{x}\right\}$. Here, $S_{0, L}$ is the Voronoi cell of $\breve{\boldsymbol{a}}_{L}=\mathbf{0}$. Xu (2006) pointed out that it is rather difficult to compute the integral of (41) since the Voronoi cell $S_{0, L}$ is constructed by cutting the m-dimensional space by infinite hyper-planes and sensitive to the covariance matrix $\boldsymbol{\Sigma}_{\widehat{\boldsymbol{a}}_{L}}$. Moreover, he recognized that the Shannon's lower probabilistic bound of error can be used to compute the upper probabilistic bound of $P\left(\breve{\boldsymbol{a}}_{L}\right)$ in the case of GNSS applications as (Xu 2006),

$$
P\left(\breve{\boldsymbol{a}}_{L}\right)=\int_{S_{0, L}} p(\boldsymbol{x}) d \boldsymbol{x} \leq \int_{E_{0}} p(\boldsymbol{x}) d \boldsymbol{x}
$$


where $E_{0}=\left\{\boldsymbol{x} \mid \boldsymbol{x}^{T} \boldsymbol{\Sigma}_{\widehat{\boldsymbol{a}}_{L}}^{-1} \boldsymbol{x} \leq \chi^{2}\right\}$, and the positive constant $\chi^{2}$ satisfies the condition that the defined ellipsoid $E_{0}$ is of unit volume. The inequality (42) was re-discovered by Hassibi and Boyd (1996, 1998). For convenient expression, we denote $P_{u p}\left(\breve{\boldsymbol{a}}_{L}\right)=\int_{E_{0}} p(\boldsymbol{x}) d \boldsymbol{x}$. The most attractive feature of Shannon's upper probabilistic bound is that it does not require any knowledge about Voronoi cell $S_{0, L}$.

Since the $E_{0}$ is mathematically very simple, one can then use approximation techniques of multiple integrals to compute $P_{u p}\left(\breve{\boldsymbol{a}}_{L}\right)$. However, before the integral computation, the constant $\chi^{2}$ must be determined according to the condition of its unit volume as (Apstol 1969)

$$
\chi^{2}=\sqrt[n / 2]{\frac{n \Gamma(n / 2)}{2 \pi^{n / 2} \sqrt{\left|\boldsymbol{Q}_{\hat{\mathbf{z}}_{f}}\right|}}}=\frac{1}{\pi} \times \sqrt[n]{\frac{n^{2} \Gamma^{2}(n / 2)}{4\left|\boldsymbol{Q}_{\hat{\mathbf{z}}_{f}}\right|}}
$$

where $\Gamma$ is the gamma function which is recursively calculated using the relation $\Gamma(x+1)=x \Gamma(x), \Gamma(1 / 2)=\sqrt{\pi}$ and $\Gamma(n)=(n-1)$ !. Especially, in two-dimensional case, the analytical formula of $P_{u p}\left(\check{\boldsymbol{a}}_{L}\right)$ is easily derived

$$
P_{u p}\left(\check{\boldsymbol{a}}_{L}\right)=1-\exp \left(-\frac{\chi^{2}}{2}\right)
$$

However, it is not easy to derive the analytical formula for the high-dimensional case. In this paper, we compute the integral $P_{u p}\left(\breve{\boldsymbol{a}}_{L}\right)$ based on the Basic theorem of Monte-Carlo Integration (Weinziel 2000; Teunissen et al. 2008)

$$
\widehat{P_{u p}}\left(\check{\boldsymbol{a}}_{L}\right)=\bar{p}(\boldsymbol{x})
$$

with $\bar{p}(\boldsymbol{x})=\frac{1}{N} \sum_{i}^{N} p\left(\boldsymbol{x}_{i}\right)$ and $N$ is the number of random samples within the ellipsoid $E_{0}$. The one-standard error of this approximation integral is

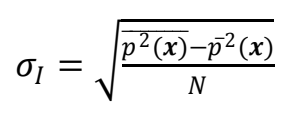

with $\overline{p^{2}(\boldsymbol{x})}=\frac{1}{N} \sum_{i}^{N} p^{2}\left(\boldsymbol{x}_{i}\right)$. To achieve the reliable approximation solution, the random samples must be enough. For more information about Monte-Carlo integration, one can refer to Weinziel (2000).

However, the regularized float solution is biased and we should specify the bias effect to evaluate the success probability of regularized AR. Let the regularized AR by regularizing ambiguities be a case study, its distribution reads $\widehat{\boldsymbol{a}}_{R A} \sim N\left(\overline{\boldsymbol{a}}+\boldsymbol{g}_{\widehat{\boldsymbol{a}}_{\boldsymbol{R} A}} \quad \boldsymbol{\Sigma}_{\widehat{\boldsymbol{a}}_{\boldsymbol{R} A}}\right)$. In this case, the PDF $p(\boldsymbol{x})$ in Eq.(42) becomes

$$
p(\boldsymbol{x})=\left|2 \pi \boldsymbol{\Sigma}_{\widehat{\boldsymbol{a}}_{R A}}\right|^{-1 / 2} \exp \left\{-\frac{1}{2}\left(\boldsymbol{x}-\boldsymbol{g}_{\widehat{\boldsymbol{a}}_{R A}}\right)^{T} \boldsymbol{\Sigma}_{\widehat{\boldsymbol{a}}_{R A}}^{-1}\left(\boldsymbol{x}-\boldsymbol{g}_{\widehat{a}_{R A}}\right)\right\}
$$

In theory, we cannot guarantee that the Shannon's upper probabilistic bound, i.e., inequality (41), holds true for any $\boldsymbol{g}_{\widehat{a}_{\boldsymbol{R A}}}$ unless the biases are small, which means that the success probability of regularized AR may be conservatively evaluated. Fortunately, in the GNSS case, the regularized biases are often rather small, referring to the section 7 for real GPS experiments. In following, we call the Shannon's upper probabilistic bound directly as success probability 
without confusion.

\subsection{Demonstration of higher success probability of regularized AR through random simulations}

In general, the regularization can effectively alleviate the model's ill-condition and thus improve the accuracies of float ambiguities as well as decrease their correlation. In such a situation, if the effect of regularized bias is ignored, the success probability of the regularized AR is definitely larger than that of LS AR, which was proven by Gui and Han (2007). However the regularized solution is biased and the bias will decrease the success probability. In other words, the regularization makes the shape of PDF of float ambiguities sharper but the bias introduces a translation to the PDF. Both factors affect the success probability, thus it is rather difficult (almost impossible) to explicitly prove the higher success probability of regularized AR if taking the bias into account.

For intuitive illustration of the improvement of regularized AR, we study two examples firstly. (i) Supposing a LS float ambiguity and its corresponding regularized solution are $\hat{a}_{L} \sim N\left(\begin{array}{lll}0 & 0.25\end{array}\right)$ and $\hat{a}_{R} \sim N\left(\begin{array}{ll}0.1 & 0.09\end{array}\right)$, their success probabilities are $68.27 \%$ and $88.60 \%$ respectively. (ii) The second one is for a two-dimensional AR case. The LS and regularized float ambiguities are normally distributed, $\left[\begin{array}{l}\hat{a}_{L, 1} \\ \hat{a}_{L, 2}\end{array}\right] \sim N\left(\left[\begin{array}{l}0 \\ 0\end{array}\right]\left[\begin{array}{ll}0.16 & 0.02 \\ 0.02 & 0.05\end{array}\right]\right)$ and $\left[\begin{array}{l}\hat{a}_{R, 1} \\ \hat{a}_{R, 2}\end{array}\right] \sim N\left(\left[\begin{array}{l}0.02 \\ 0.03\end{array}\right] \quad\left[\begin{array}{ll}0.090 & 0.012 \\ 0.012 & 0.040\end{array}\right]\right)$, respectively. We compute their success probabilities based on Monte-Carlo integration formulae (45) and (46) with $N=10^{5}$. The success probabilities are $83.85 \%$ and $93.08 \%$ and their approximation accuracies are $0.15 \%$ and $0.2 \%$, respectively. From these two examples, we conclude that if the PDF of regularized float ambiguities becomes sufficiently sharper and the regularized biases retain relatively small values, the success probability as the integral of PDF over the pull-in region is hardly affected (see also Teunissen 2001), which is the often case for the fast GNSS AR situations. In addition, the success probability of the two-dimensional LS solution is re-computed by (44). The result is $83.89 \%$ which is very close to the result from the Monte-Carlo method. It implies that the Monte-Carlo integration can reliably approximate the integral of (42) if the random samples are sufficient.

Higher success probabilities of the regularized AR are now numerically demonstrated by random simulations which allow ambiguity normal equations to vary with different degrees of ill-condition and to be solved by using regularization and LS methods respectively. The random simulations are implemented in this paper mainly according to Xu (2001), Chang et al. (2005) and Li and Shen (2010). The float ambiguity vector $\widehat{\boldsymbol{a}}_{L}$ is firstly generated by $\widehat{\boldsymbol{a}}_{L}=$ $\frac{\sigma_{C}}{\lambda} \times \operatorname{randn}(m, 1)$, where $\operatorname{randn}(m, 1)$ is a Matlab built-in function to generate a vector of $m$ random elements of standard normal distribution. The normalized orthogonal matrix $\boldsymbol{V}$ is computed by factorization of a random square matrix which also generated by Matlab function. The eigenvalues $\theta_{i}(i=1,2, \ldots, m)$ of the diagonal matrix $\boldsymbol{\Theta}$ are the positive simulated random numbers. Then the simulated normal equations consisting of $N_{L}$ and $u$ are trivially determined by 
$\boldsymbol{N}_{L}=\boldsymbol{V} \boldsymbol{\Theta} \boldsymbol{V}^{T}$ and $\boldsymbol{u}=\boldsymbol{N}_{L} \times \widehat{\boldsymbol{a}}_{L}$. It is crucial to apply a scale factor, for instance $10^{5}$, to the last three eigenvalues for obtaining three small eigenvalues and thus a gap between them and the others (Teunissen and Kleusberg 1998; Li and Shen 2010). In order to fully demonstrate the performance of regularized AR, three scale factors (SF), i.e. $10^{4}, 10^{5}$ and $10^{6}$, are applied to describe three different ill-posed degrees of ambiguity normal equations.

We adopt regularization and LS methods to solve the ambiguities with the following steps:

(i) The RP is computed by Eq.(34) with the cofactor matrix of initial ambiguities $\boldsymbol{Q}_{\widehat{a}}=\frac{\boldsymbol{\sigma}_{C}^{2}}{\lambda^{2} \times \sigma_{0}^{2}}$.

(ii) The LS float solution $\left(\widehat{\boldsymbol{a}}_{L}, \boldsymbol{\Sigma}_{\widehat{\boldsymbol{a}}_{L}}\right)$ and the regularized float solution $\left(\widehat{\boldsymbol{a}}_{R A}, \boldsymbol{\Sigma}_{\widehat{\boldsymbol{a}}_{R A}}\right)$ are computed by Eqs.(16) and (31), respectively.

(iii) The regularized bias $\widehat{\boldsymbol{g}_{\widehat{\boldsymbol{a}}_{R A}}}$ is computed by substituting the regularized float solution $\widehat{\boldsymbol{a}}_{R A}$ into Eq.(32).

(iv) The success probabilities of regularized and LS AR are computed based on Eq. (45) with the corresponding PDFs and $N=10^{5}$.
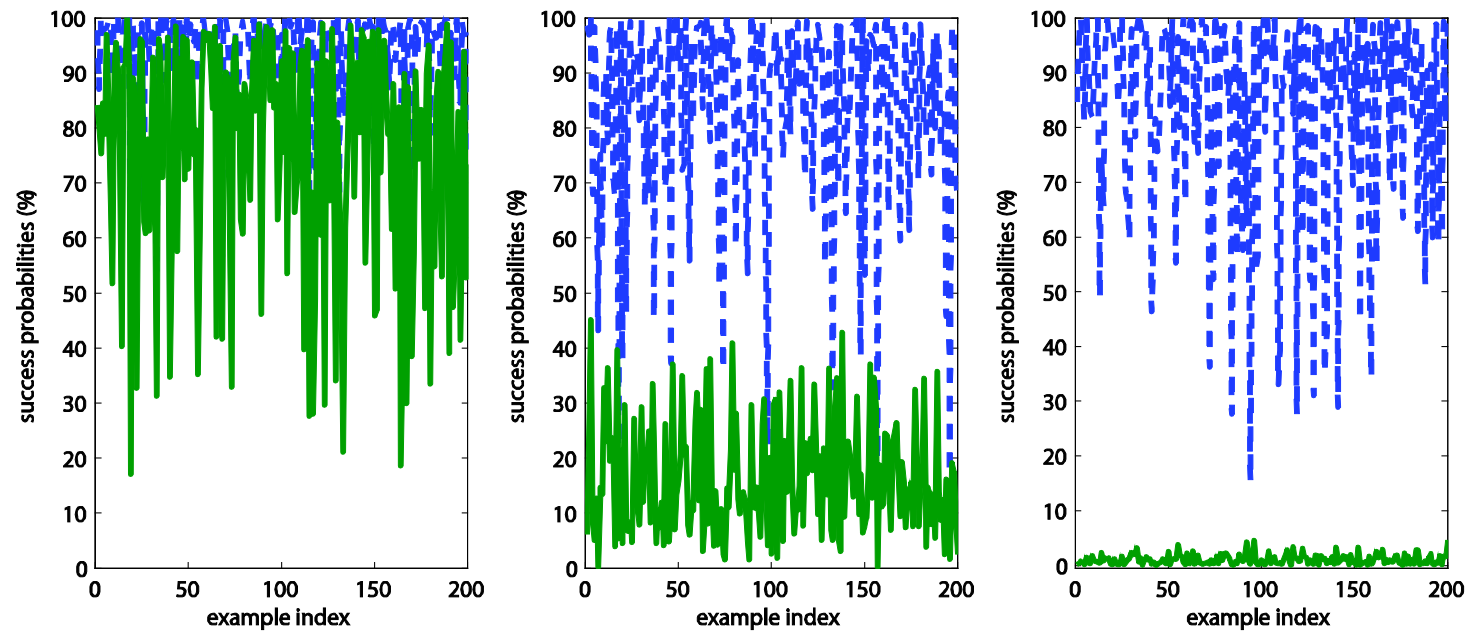

Figure 2. Success probabilities of ambiguity resolution $\left(\sigma_{C}=0.3, m=5\right.$; left: $\mathrm{SF}=10^{-4}$, middle: $\mathrm{SF}=10^{-5}$, right: $\left.\mathrm{SF}=10^{-6}\right)$
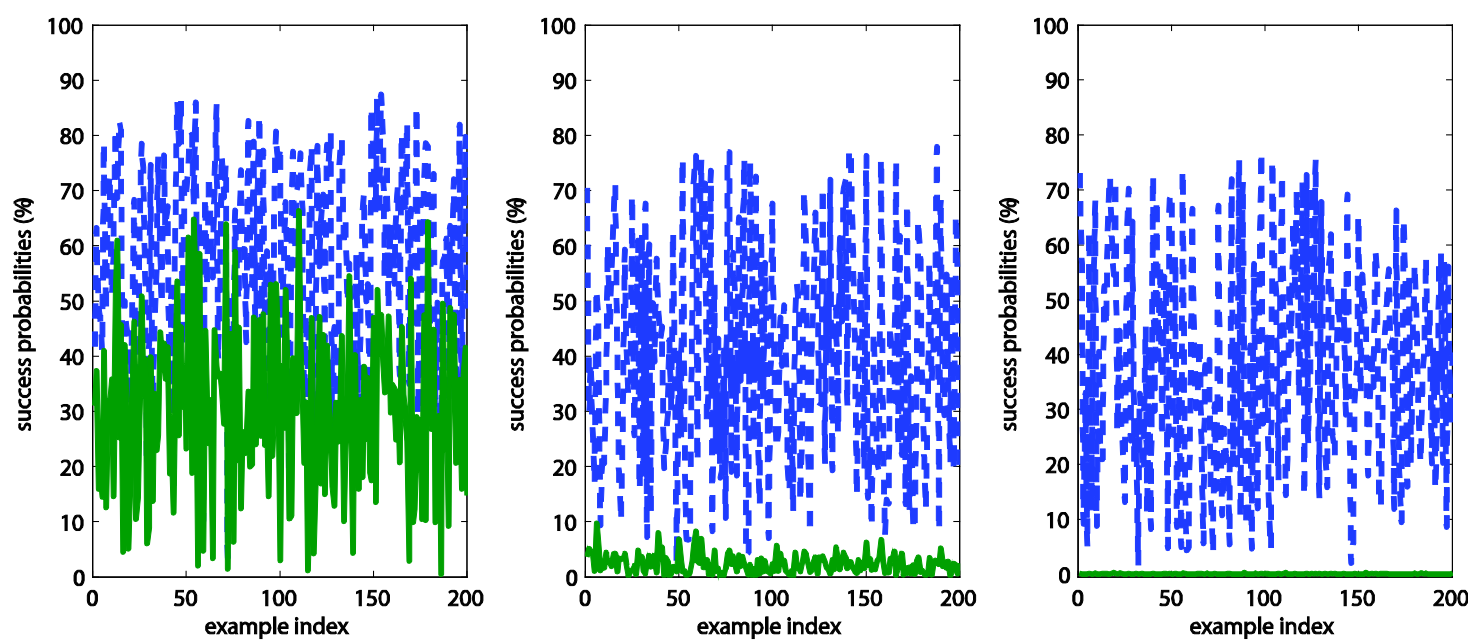

Figure 3. Success probabilities of ambiguity resolution $\left(\sigma_{C}=0.5, m=5\right.$; left: $\mathrm{SF}=10^{-4}$ middle: $\mathrm{SF}=10^{-5}$, right: $\left.\mathrm{SF}=10^{-6}\right)$ 
Table 1: Success probabilities of regularized and LS AR (\%)

\begin{tabular}{rrrrrrr}
\hline \multirow{2}{*}{$\sigma_{C}(\mathrm{~m})$} & \multicolumn{3}{c}{ Regularized AR } & \multicolumn{3}{c}{$\mathrm{LS}$ AR } \\
\cline { 2 - 7 } & $\mathrm{SF}=10^{-4}$ & $\mathrm{SF}=10^{-5}$ & $\mathrm{SF}=10^{-6}$ & $\mathrm{SF}=10^{-4}$ & $\mathrm{SF}=10^{-5}$ & $\mathrm{SF}=10^{-6}$ \\
\hline 0.3 & 93.84 & 83.54 & 82.49 & 75.58 & 16.43 & 1.01 \\
0.4 & 78.49 & 63.24 & 62.79 & 51.53 & 6.00 & 0.31 \\
0.5 & 57.47 & 39.96 & 37.73 & 30.17 & 2.40 & 0.09 \\
\hline
\end{tabular}

The results are presented in Figure 2 for $\sigma_{C}=0.3 \mathrm{~m}$ and $m=5$ and Figure 3 for $\sigma_{C}=0.5 \mathrm{~m}$ and $m=5$, respectively. For each figure, three subplots from left to right reflect the SFs of $10^{-4}, 10^{-5}$ and $10^{-6}$ assigned in the simulations, respectively. The dash-line and the solid-line represent the success probabilities of regularized and LS solutions, respectively. For the larger SF pertaining to a slight ill-condition, the larger success probabilities are obtained in both regularization and LS solutions, vice versa. However, the regularization can always improve the success probability, albeit the improvement degree depends on the ill-conditioned degree of the model. Comparing Figures 2 and 3, it is observed that the pseudorange accuracy (i.e., accuracy of initial ambiguity) is also an important factor for AR. To verify this point, we conduct more simulations for different pseudorange accuracies. The results are shown in Table 1. Apparently, the success probabilities are affected by both the ill-conditioned degree of the model and the pseudorange accuracy though their effect degrees are different. However, when the model is severely ill-conditioned and this ill-condition is not efficiently mitigated, the precise pseudoranges can hardly improve the successful AR. For example, in the case of $\mathrm{SF}=10^{-6}$ (see the last column of Table 1 which corresponds to the most severely ill-conditioned models), the success probabilities are almost zero although when $\sigma_{C}=0.3 \mathrm{~m}$. Up to now, we have numerically demonstrated the higher success probability of regularized AR by random simulations.

\section{Performance of regularized AR using real GPS observations}

We further evaluate the superior performance of regularized AR with respect to ILS solutions using a real GPS data set. The float solutions are solved by using LS and regularization respectively and then LAMBDA method is employed to fix their integer solutions. Total 8000 epochs of single frequency data are collected for a 4.6km baseline using the Leick MC500 receivers with the sample interval of $1 \mathrm{~s}$. The observation types include C1 pseudorange and L1 phase and their accuracies are assessed to be $0.345 \mathrm{~m}$ and $3.1 \mathrm{~mm}$ according to $\mathrm{Li}$ et al. (2008). The elevation mask is set to 13 degrees. Figure 4 illustrates the PDOP series (A) and the number of observed satellites (B) over the whole observation period. 

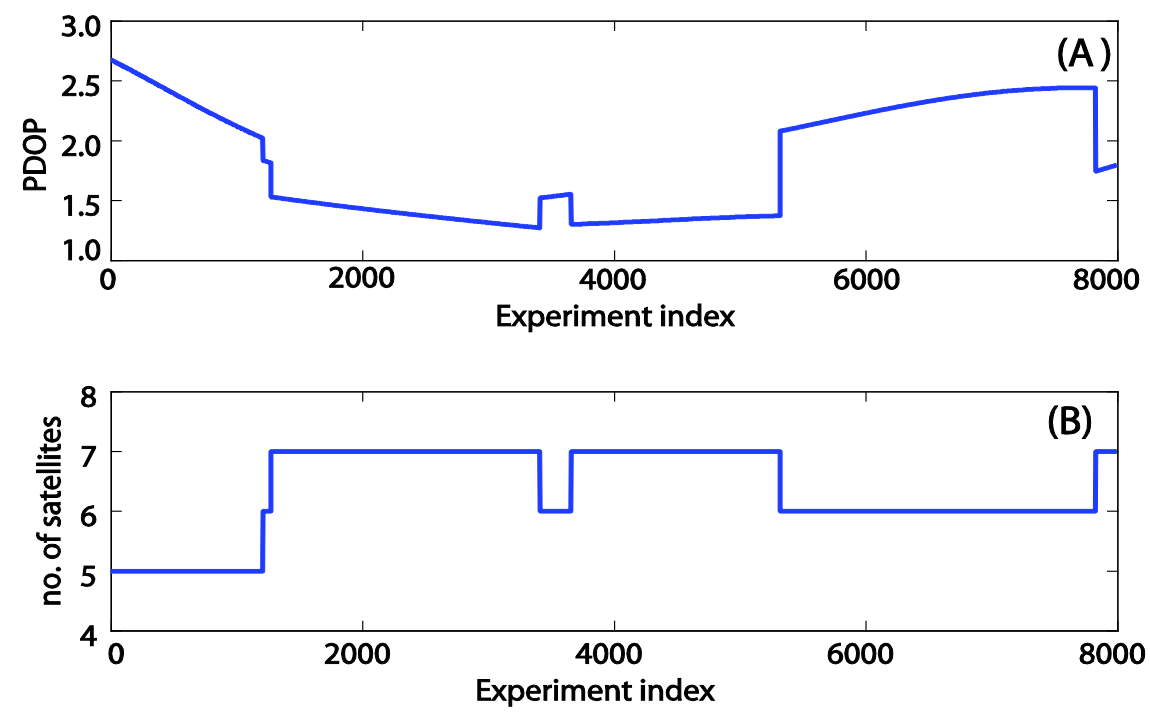

Figure 4. The PDOP series (A) and the number of observed satellites (B) in the whole observation span

First of all, all ambiguities are correctly fixed using all observations serving as true integers in the following analysis. We statistically compute the success probabilities of correct AR as

$$
P=n_{\text {cor }} / n_{\text {tot }} \times 100 \%
$$

where $n_{\text {cor }}$ and $n_{\text {tot }}$ are the number of computations with all ambiguities being correctly fixed and the number of total computations. In addition, to understand the overall quality of the float ambiguities, we define the mean accuracy level as

$$
\sigma_{\text {mean }}=\sqrt{\operatorname{tr}\left(\boldsymbol{\Sigma}_{\widehat{a}}\right) / m}
$$

where $\boldsymbol{\Sigma}_{\widehat{a}}$ is the covariance matrix for the LS float ambiguities and it is replaced by the MSE matrix $\boldsymbol{M}_{\widehat{a}}$ for the regularized float ambiguities including the effects of regularized biases; $m$ is the number of ambiguities. In fact it reveals a mean accuracy level of a set of float ambiguities. In the following, we will evaluate the performances of two regularized AR schemes, which are specified by regularizing baseline and ambiguity parameters respectively with respect to those of LS AR.

\subsection{Regularized AR by regularizing baseline parameters}

Two experimental schemes are carried out to demonstrate the regularized AR performance, which correspond to use of the geometry-based and time-averaged models in computing initial baseline respectively, as shown in Table 2.

In this subsection, all experiments use both L1 phase and C1 pseudorange to form the error equations (14) and $\kappa=10000$. In each computation, a moving window of 10 epoch data are used, and the moving window moves forward one epoch for the next computation. For the RB_GB scheme, Figure 5 presents the computed RP for all computations. Referring to Figure 4, these RPs strongly depend on the PDOP values. A smaller PDOP is corresponding to a larger RP. 
It is because the uncertainty of initial baseline computed from pseudoranges based on a geometry-based model is lower as the PDOP is smaller. Thus a lager RP brings the regularized solution closer to precise initial values. Conversely, when the PDOP is larger, the uncertainty of computed initial baseline will be larger, and the estimated RP is automatically smaller so as to reduce the dependence of regularized solution on the initial value. Figure 6 shows the conditional numbers of LS and regularized normal equations. It is evident that the conditional numbers are significantly reduced by regularization, which means that the regularization can indeed effectively reduce the model's ill-condition.

Table 2: Two experiment schemes for regularized AR by regularizing baseline parameters (RB_GB denotes computing the initial baseline based on geometry-based model while RB_TA based on time-averaged model)

\begin{tabular}{|c|c|c|}
\hline & RB_GB & RB_TA \\
\hline Float solution & \multicolumn{2}{|c|}{$\widehat{\boldsymbol{a}}_{R C}=\boldsymbol{N}_{\boldsymbol{a \boldsymbol { a }}}^{-1}\left(\boldsymbol{u}_{a}-\boldsymbol{N}_{a b} \widehat{\boldsymbol{b}}_{R C}\right), \boldsymbol{\Sigma}_{\widehat{\boldsymbol{a}}_{R C}}=\sigma_{0}^{2}\left(\boldsymbol{N}_{a a}-\boldsymbol{N}_{a b} \widetilde{\boldsymbol{N}}_{b b}^{-1} \boldsymbol{N}_{b a}\right)^{-1}$} \\
\hline Bias & \multicolumn{2}{|c|}{$\widehat{\boldsymbol{g}_{\widehat{a}_{R C}}}=\boldsymbol{N}_{a a}^{-1} \boldsymbol{N}_{a b} \boldsymbol{g}_{\widehat{\boldsymbol{b}}_{R C}}=\alpha \boldsymbol{N}_{a a}^{-1} \boldsymbol{N}_{a b} \widetilde{\boldsymbol{N}}_{b \mid a}^{-1} \widehat{\boldsymbol{b}}_{R C}$} \\
\hline $\mathrm{RP}$ & \multicolumn{2}{|c|}{$\alpha=\arg \min _{\alpha>0} \operatorname{tr}\left(\left(\boldsymbol{N}_{a a}-\boldsymbol{N}_{a b} \widetilde{\boldsymbol{N}}_{b b}^{-1} \boldsymbol{N}_{b a}\right)^{-1}+\alpha^{2} \boldsymbol{N}_{a a}^{-1} \boldsymbol{N}_{a b} \widetilde{\boldsymbol{N}}_{b \mid a}^{-1} \boldsymbol{Q}_{\widehat{\boldsymbol{b}}} \widetilde{\boldsymbol{N}}_{b \mid a}^{-1} \boldsymbol{N}_{b a} \boldsymbol{N}_{a a}^{-1}\right)$} \\
\hline$Q_{\widehat{b}}$ & $\kappa \times\left(\sum_{j=1}^{n} \boldsymbol{B}_{j}^{T} \boldsymbol{Q}_{\boldsymbol{\Phi}_{j}}^{-1} \boldsymbol{B}_{j}\right)$ & $\frac{\kappa}{n} \times\left(\overline{\boldsymbol{B}}^{T} \boldsymbol{Q}_{\bar{\Phi}}^{-1} \overline{\boldsymbol{B}}\right)^{-1}$ \\
\hline
\end{tabular}

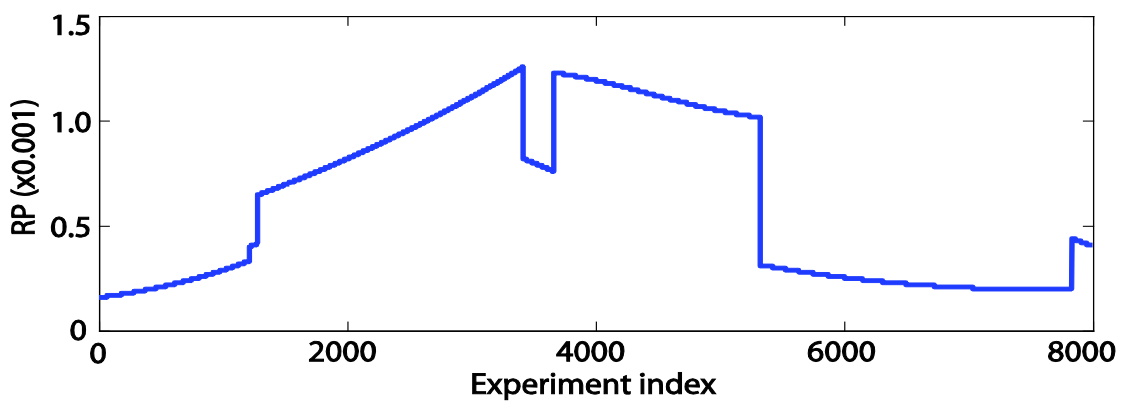

Figure 5. Computed regularization parameters

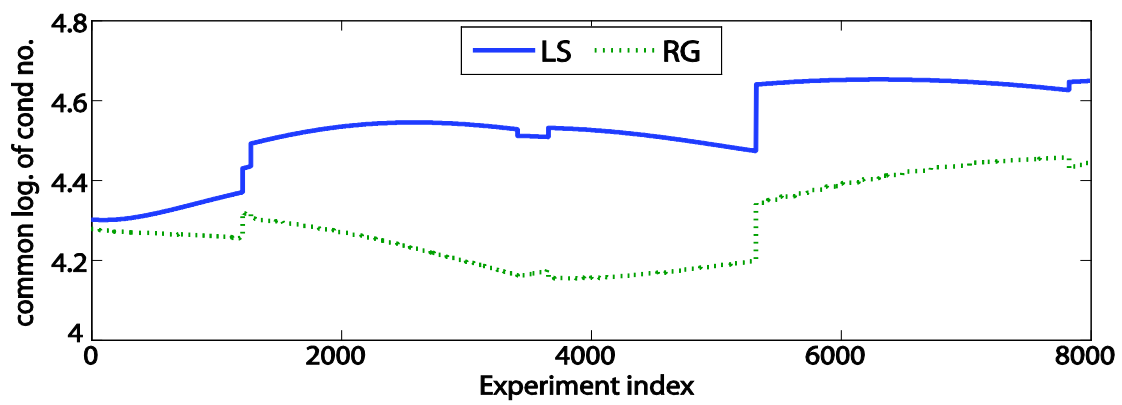

Figure 6. Conditional numbers of LS and regularized normal equations

The differences between the LS and regularized float ambiguities and their true values are shown in Figure 7. The differences of LS solutions are similar to those of the regularized solutions in the RB_GB scheme and all smaller than 
3 cycles. The theoretical accuracy of these differences is computed by $\sqrt{\sum_{i=1}^{n} \delta a_{i}^{2} / n}$ to be 0.69 cycles for LS and regularization, where $\delta a_{i}$ is the ith difference and $n$ is the number of differences. It means that for a good estimation, the mean accuracy computed by Eq.(49) from the covariance matrix of float ambiguities should be close to this theoretical accuracy. Figure 8 shows the mean accuracies of LS and regularized float solutions computed by Eq.(49), and their means are 1.10 cycles and 0.88 cycles for LS and regularization, respectively. Obviously, the mean accuracies of regularized float ambiguities are closer to the theoretical accuracy than those of LS float ambiguities. Therefore, the covariance matrix of regularized float ambiguities can reflect their real accuracies. In other words, the regularized float solutions are admissible with their covariance matrix better than the LS solutions.
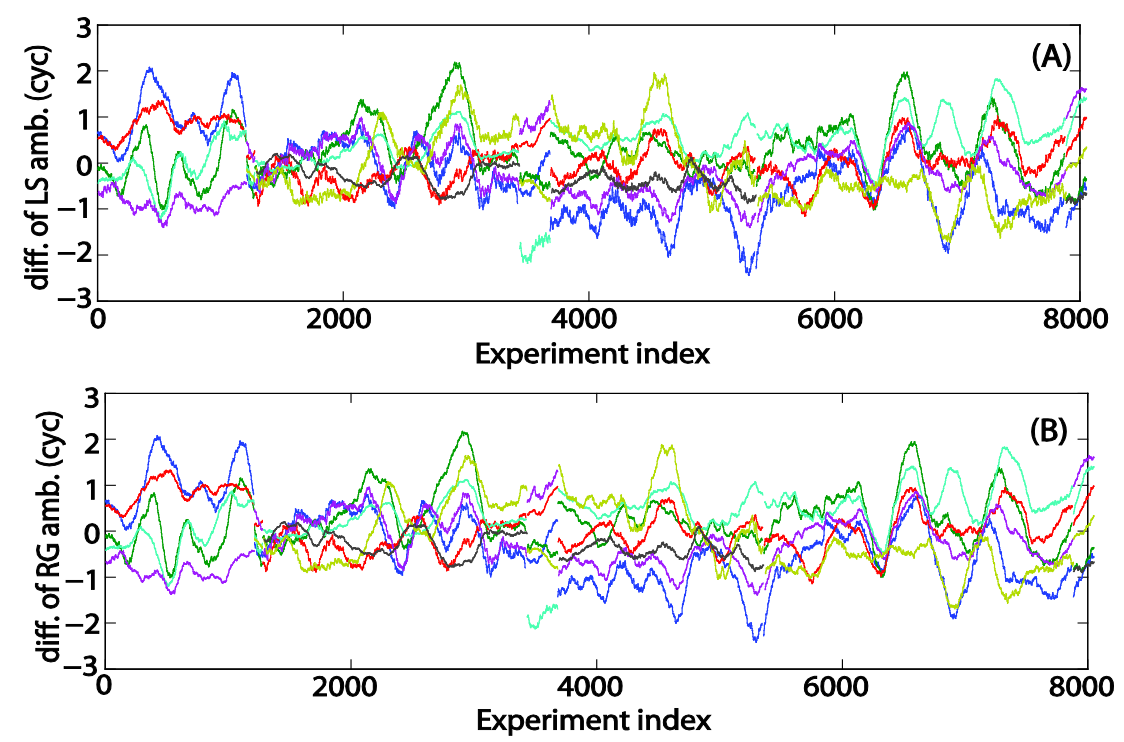

Figure 7. Differences between true ambiguities and LS (A) as well as regularized float ambiguities (B)

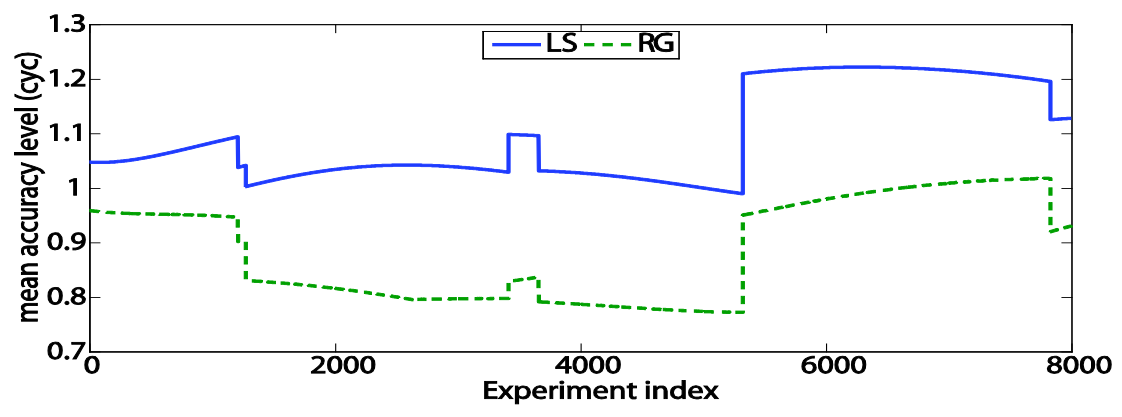

Figure 8. Mean accuracies of LS and regularized float ambiguities

Because the regularized float solution is biased and the LAMBDA method is used to fix ambiguity, the decorrelated regularized biases may affect AR when they are sufficiently large. The biases of the regularized float ambiguities are computed according to the formula in Table 2, and then they are transformed by multiplying the Z-transformation ma- 
trix output from LAMBDA method to obtain the decorrelated regularized biases. As shown in Figure 9, fortunately, these biases are mostly smaller than 0.01 cycles in magnitude and ignorable comparing with the accuracy improvement by regularization (see also Figure 8). In fact, the regularization makes the PDF of float ambiguities more peaked such that these small regularized biases result in very little change to the success probability (Teunissen 2001). However, if the biases turn so large to affect the correct AR, additional process may be needed. A possible bias-corrected approach for regularized solution is motivated by Xu et al. (2006).

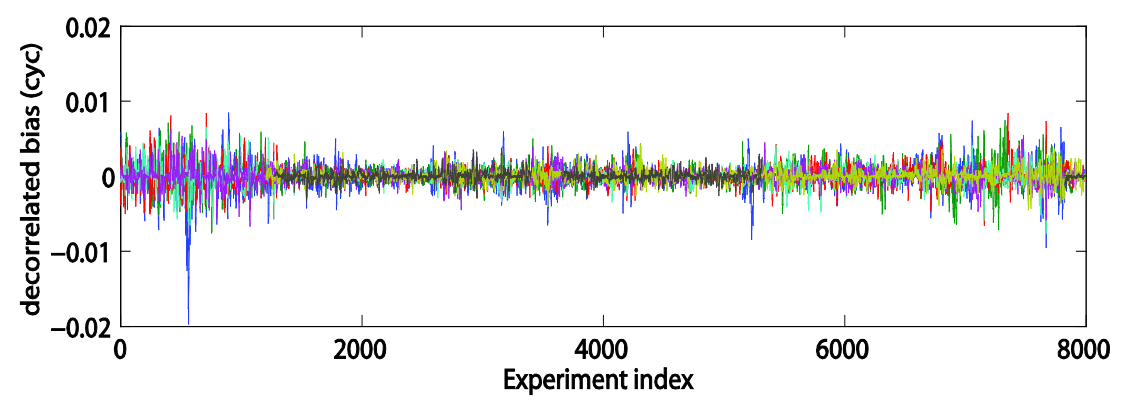

Figure 9. Decorrelated biases of regularized float ambiguities

A further assessment of the regularized AR with RB_GB and RB_TA models is carried out to explore its dependence on the numbers of data epochs with respect to the performance of ILS AR. The success probabilities computed by Eq. (48) are shown in Table 3. Results from RB_GB and RB_TA are quite consistent with each other, which imply that the time-averaged model is a good alternative to the geometry-based model to simplify the computation when the sample interval is small. With accumulation of continuous observations in the linear equation system, the success probabilities for both regularized and LS are increased and the advantage of regularization over LS is decreased, because the model's ill-condition degree is reduced and the LS solution is stabilized with more measurements. In other words, the regularization would be no longer required when the observation accumulation is sufficient to make the underlying model strong enough.

Table 3: Success probabilities of regularized AR by regularizing the baseline parameters (\%)

\begin{tabular}{cccccccccc}
\hline \multirow{2}{*}{ Schemes } & \multicolumn{8}{c}{ Number of data epochs } \\
\cline { 2 - 10 } & 1 & 3 & 5 & 10 & 30 & 60 & 90 & 120 & 150 \\
\hline LS & 63.76 & 65.15 & 65.47 & 66.02 & 69.36 & 73.20 & 79.97 & 82.61 & 85.89 \\
RB_GB & 70.01 & 70.76 & 71.22 & 71.38 & 74.39 & 78.30 & 83.43 & 85.18 & 88.47 \\
RB_TA & 70.01 & 70.76 & 71.22 & 71.38 & 74.38 & 78.30 & 83.43 & 85.18 & 88.47 \\
\hline
\end{tabular}




\subsection{Regularized AR by regularizing ambiguity parameters}

Three experiment schemes are designed to demonstrate the regularized AR by regularizing ambiguity parameters as outlined in Table 4. Similar to the subsection 7.1, three different schemes are specified to compute initial ambiguities using the geometry-based (RA_GB), time-averaged (RA_TA) and geometry-free (RA_GF) models, respectively, in which both L1 phase and C1 pseudorange are used in RA_GB and RA_TA, whereas only L1 phase is used in RA_GF.

Table 4: Three experiment schemes for regularized AR by regularizing ambiguity parameters (RA_GB, RA_TA and RA_GF denote to compute the initial ambiguities based on the geometry-based, time-averaged and geometry-free models, respectively)

\begin{tabular}{|c|c|c|c|}
\hline & RA_GB & RA_TA & RA_GF \\
\hline Float solution & \multicolumn{3}{|c|}{$\widehat{\boldsymbol{a}}_{R A}=\widetilde{\boldsymbol{N}}_{\boldsymbol{a} \mid \boldsymbol{b}}^{-1} \boldsymbol{u}_{\boldsymbol{a} \mid \boldsymbol{b}} ; \boldsymbol{\Sigma}_{\widehat{\boldsymbol{a}}_{R A}}=\sigma_{0}^{2} \widetilde{\boldsymbol{N}}_{\boldsymbol{a} \mid \boldsymbol{b}}^{-1} \boldsymbol{N}_{a \mid b} \widetilde{\boldsymbol{N}}_{\boldsymbol{a} \mid \boldsymbol{b}}^{-1}$} \\
\hline Bias & \multicolumn{3}{|c|}{$\widehat{\boldsymbol{g}_{\widehat{\boldsymbol{a}} R A}}=-\alpha \widetilde{\boldsymbol{N}}_{a \mid b}^{-1} \widehat{\boldsymbol{a}}_{R A}$} \\
\hline $\mathrm{RP}$ & \multicolumn{3}{|c|}{$\alpha=\operatorname{argmin}_{\alpha>0} \operatorname{tr}\left(\widetilde{\boldsymbol{N}}_{\boldsymbol{a} \mid \boldsymbol{b}}^{-1}\left(\boldsymbol{N}_{a \mid b}+\alpha^{2} \boldsymbol{Q}_{\widehat{\boldsymbol{a}}}\right) \widetilde{\boldsymbol{N}}_{\boldsymbol{a} \mid \boldsymbol{b}}^{-1}\right)$} \\
\hline \multirow{2}{*}{$Q_{\widehat{a}}$} & $\boldsymbol{Q}_{\boldsymbol{\Phi}} / n+\kappa \overline{\boldsymbol{B}}\left(\sum_{j=1}^{n} \boldsymbol{B}(j)^{T} \boldsymbol{Q}_{\boldsymbol{\Phi}}^{-1} \boldsymbol{B}(j)\right)^{-1} \overline{\boldsymbol{B}}^{T}$ & $\underline{\boldsymbol{Q}_{\boldsymbol{\Phi}}+\kappa \overline{\boldsymbol{B}}\left(\overline{\boldsymbol{B}}^{T} \boldsymbol{Q}_{\boldsymbol{\Phi}}^{-1} \overline{\boldsymbol{B}}\right)^{-1} \overline{\boldsymbol{B}}^{T}}$ & $\underline{(1+\kappa) \boldsymbol{Q}_{\boldsymbol{\Phi}}}$ \\
\hline & $\lambda^{2}$ & $\lambda^{2} \times n$ & $\overline{\lambda^{2} \times n}$ \\
\hline
\end{tabular}

Because the performance of RA_GB and RA_TA are similar to those of RB_GB and RB_TA given in previous subsection, only the performance of RA_GF is demonstrated. Again, a moving window of 10 consecutive data epochs is used in each computation. Figure 10 gives the computed regularized parameters which are distinctly different from those in Figure 5, because the PDOP is free of influence on the geometry-free model and only pseudorange accuracy dominates the initial ambiguities. Figure 11 presents the conditional numbers of LS and regularized normal equations. The mean conditional number can be reduced from $10^{7.7}$ to $10^{4.6}$ when the regularization is applied. Figure 12 shows the differences between the LS and regularized float ambiguities and their true values respectively. The differences are in the range of tens of cycles for the LS solution and reduced to smaller than 3 cycles for regularization. Comparing with Figure 7, the LS solution of RA_GF is much worse mainly since only phase data are used and the derived normal equations are severely of ill-condition.

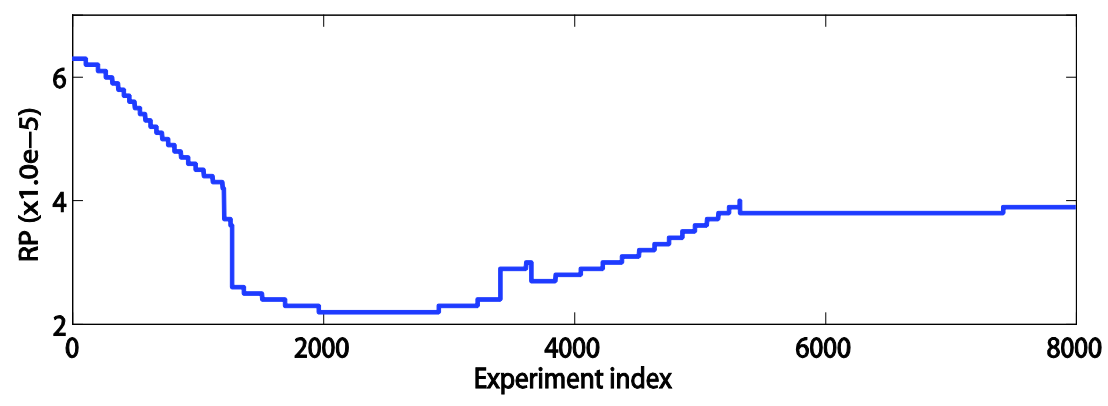

Figure 10. Computed regularization parameters 


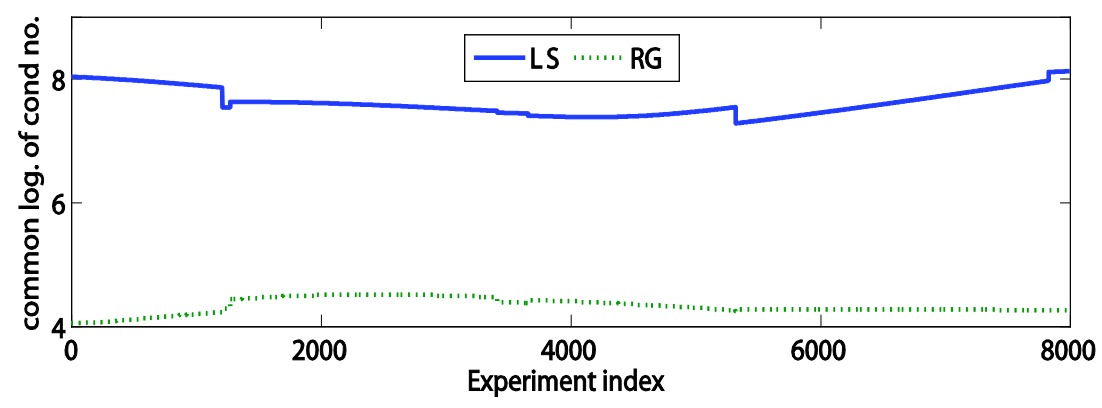

Figure 11. Conditional numbers of normal equations of LS and regularization

Figure 13 shows the decorrelated regularized biases. Although the values are larger than those shown in Figure 9, they are still small enough comparing to the accuracy improvement after regularization. Figure 14 illustrates the mean accuracies of LS and regularized float ambiguities, where the y axis denotes the common logarithm of the mean accuracies. The average of these mean accuracies of LS float ambiguities is 35.24 cycles, but the regularization improves it to 0.75 cycles.
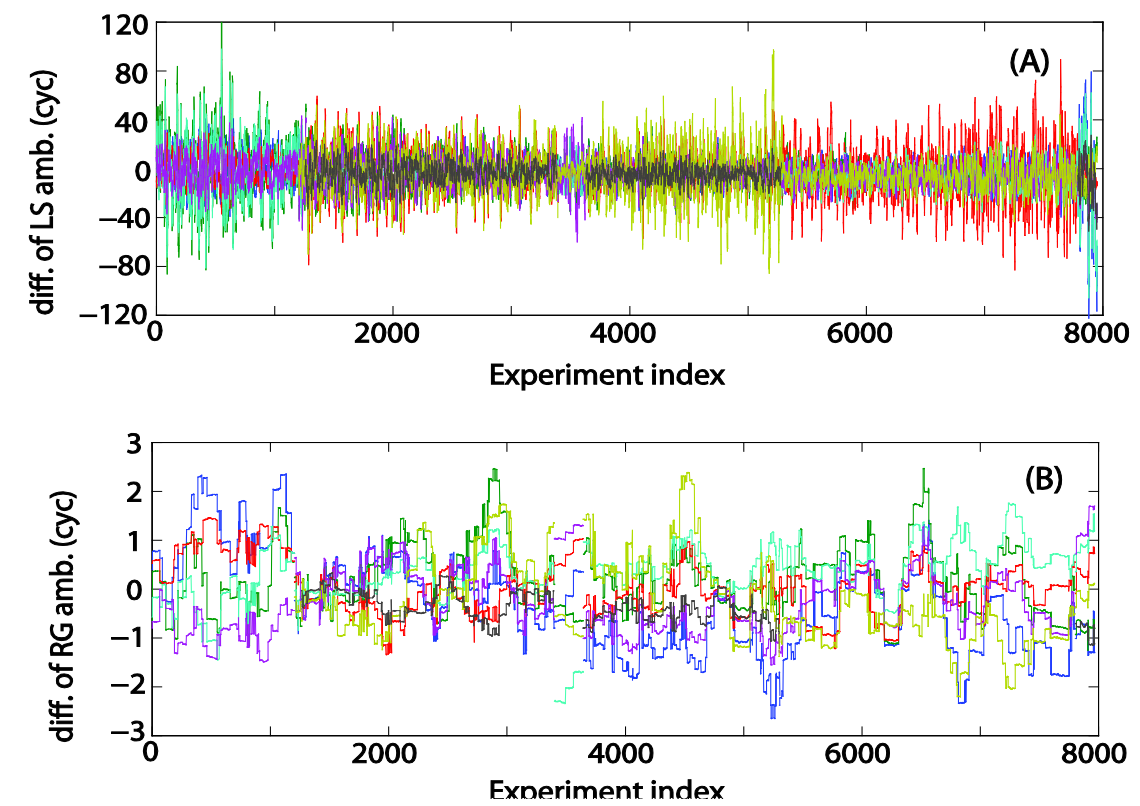

Figure 12. Differences between true ambiguities and LS (A) ambiguities as well as regularized ambiguities (B)

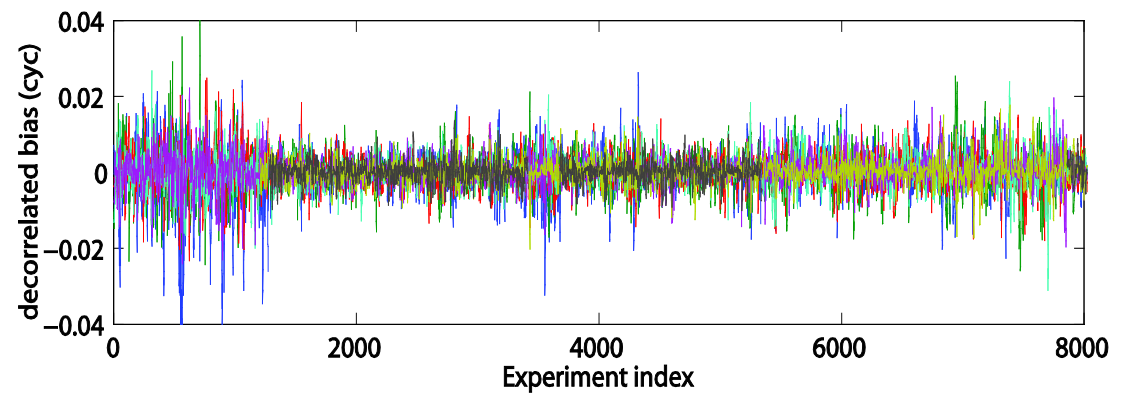

Figure 13. Decorrelated biases of regularized ambiguities 


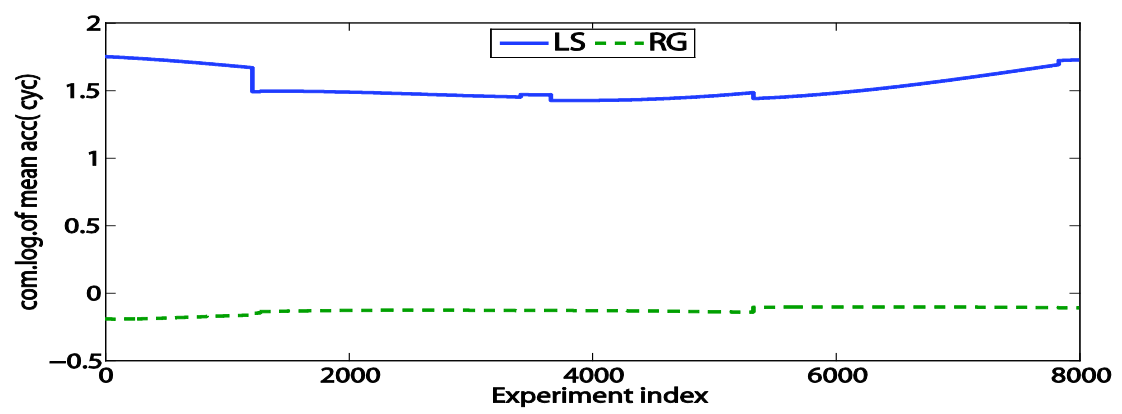

Figure 14. Mean accuracies of LS and regularization

We also assess performances of three regularized AR schemes to shown their dependence on the different numbers of data epochs. The success probabilities computed by Eq.(48) are given in Table 5. The performances of RA_GB and RA_TA are consistent with RB_GB and RB_TA, referring to Table 3, because the essences of computing the initial baselines and ambiguities are same. Although the success probabilities of RA_GF is lower than those of the other schemes, but its improvements are more significant with respect to the LS performance.

Table 5: Success probabilities of regularized AR by regularizing ambiguity parameters (\%)

\begin{tabular}{cccccccccc}
\hline \multirow{2}{*}{ Schemes } & \multicolumn{10}{c}{ Number of data epochs } \\
\cline { 2 - 10 } & 1 & 3 & 5 & 10 & 30 & 60 & 90 & 120 & 150 \\
\hline LS & 63.76 & 65.15 & 65.47 & 66.02 & 69.36 & 73.20 & 79.97 & 82.61 & 85.89 \\
RA_GB & 73.30 & 74.03 & 74.37 & 74.77 & 77.10 & 81.39 & 85.36 & 87.09 & 89.43 \\
RA_TA & 73.30 & 74.03 & 74.37 & 74.77 & 77.10 & 81.39 & 85.36 & 87.11 & 89.44 \\
\hline LS & - & 0.11 & 1.03 & 2.44 & 10.34 & 22.08 & 34.67 & 46.55 & 56.39 \\
RA_GF & - & 59.51 & 59.44 & 60.13 & 62.77 & 63.73 & 68.81 & 75.17 & 79.08 \\
\hline
\end{tabular}

\section{Summary and remarks}

In this paper, we have comprehensively addressed the fast GNSS AR as an ill-posed problem. The key has been to determine the reasonable RP where the true values of unknowns are necessary in theory but impossible to obtain in practice. Consequently, the paper has proposed to replace the quadratic matrix of the true values of unknowns with the covariance matrix of their initial values. As a result, the problem of computing a reasonable RP is turned to computing the reliable initial values of unknowns and the derived RP depends on the precision of initial values. Normally a larger RP corresponds to more precise initial values and brings the regularized solution closer to the initial values. Moreover, two regularization AR schemes have been examined, namely by regularizing the baseline and ambiguity parameters, respectively. For each scheme, the different models for computing initial values were specified.

Experiment studies with specially designed random simulations have numerically demonstrated that the higher 
success probabilities are generally achievable with regularized AR with respect to the LS AR. Furthermore using a real GPS data set, the regularized AR has also demonstrated superior performance in actual success probability with respect to the LS AR method.

In general, the regularization can effectively mitigate the model's ill-condition and then stabilize the float solutions. In other words, the regularized float ambiguities are closer to their integers and have a less correlated covariance matrix as compared to the LS float ambiguities. Consequently, higher success AR probability can be achieved, especially in the case of the strong ill-posed observational models with fewer observational data epochs.

Acknowledgements This work is partially supported by the National Natural Science Funds of China (Grant No. 40874016) and partially supported by the fund from the Key Laboratory of Advanced Surveying Engineering of SBSM (Grant No. TJES0809). The authors also acknowledge the collaborative opportunity provided through the Australian Cooperative Research Centre for Spatial Information at Queensland University of Technology.

\section{References}

Abidin H (1993) On-the-fly ambiguity resolution: formulation and results. Manuscr Geod, 18:380-405

Apostol TM (1969) Multi-variable calculus and linear algerbra, with applications to differential equations and probability. Calculus Vol.2, Second edition, Wiley, New York.

Chang X, Yang X, Zhou T (2005) MLAMBDA: a modified LAMBDA algorithm for integer least-squares estimation. J Geod 79:552-565

Chen D (1994) Development of a fast ambiguity search filtering (FASF) method for GPS carrier phase ambiguity resolution. UCGE Reports 20071, PhD dissertation

Counselman C, Gourevitch S (1981) Miniature interferometer terminals for earth surveying: ambiguity and multipath with the Global Positioning System. IEEE Trans on Geoscience and Remote Sensing 19(4):244-252

de Jonge P, Tiberius C (1996) The LAMBDA method for integer ambiguity estimation: implementation aspects, LGR-Series, Publications of the Delft Geodetic Computing Centre, No.12

Dong D, Bock Y (1989) Global Positioning System network analysis with phase ambiguity resolution applied to crustal deformation studies in California. J Geophys Res 94(B4):3949-3966

Euler H, Landau H (1992) Fast ambiguity resolution on-the-fly for real-time applications. Proc 6th Int. Geod Symp. On Satellite Positioning, Columbus, Ohio, 17-20 March, 650-659

Frei E, Beulter G (1990) Rapid static positioning based on the fast ambiguity resolution approach 'FARA': theory and first results. Manuscr Geod 15:326-356

Grafarend E (2000) Mixed integer/real valued adjustment (IRA) problems: GPS initial cycle ambiguity resolution by 
means of LLL algorithm. GPS Solut 4:31-44

Gui Q, Han S (2007) New algorithm of GPS rapid positioning based on double $k$-type ridge estimation. J Surv Eng, 133(4):173-178.

Hansen P (1992) Analysis of discrete ill-posed problems by means of the L-curve. SIAM Review 34:561-580

Hassibi A, Boyd S (1996) Integer parameter estimation in linear models with applications to GPS. Proc. IEEE Conf. Decision and Control, Kobe, Japan, pp:3245-3251

Hassibi A, Boyd S (1998) Integer parameter estimation in linear models with applications to GPS. IEEE Trans on Signal Processing 11(11):2938-2952

Hatch R (1990) Instantaneous ambiguity resolution. Proc KIS’90, Banff, Canada, 10-13 Sept, pp: 299-308

Li B, Shen Y (2008) Prior baseline information based fast GPS ambiguity resolution. Acta Geod et Cart Sin 37(4):413-417

Li B, Shen Y (2009) Fast GPS ambiguity resolution with available constraints. Geomatics and Infor Sci of Wuhan Univ 34(1):177-121.

Li B, Shen Y (2010) GNSS ambiguity resolution with constraints from normal equations. J Surv Eng 136(2):63-71

Li B, Shen Y, Xu P (2008) Assessment of stochastic models for GPS measurements with different types of receivers. Chi Sci Bull 53(20): 3219-3225

Liu L, Hsu H, Zhu Y, Ou J (1999) A new approach to GPS ambiguity decorrelation. J Geod 73:478-490

O’Keefe K, Petovello M, Lachapelle G, Cannon M (2007) Assessing probability of correct ambiguity resolution in the presence of time-correlated errors, Navigation, 53 (4): 269-282

Ou J, Wang Z (2004) An improved regularization method to resolve integer ambiguity in rapid positioning using single frequency GPS receivers. Chi Sci Bull 49(2):196-200

Park C, Kim I, Lee J, Jee G (1996) Efficient ambiguity resolution using constraint equation. Proc PLANS96, IEEE, $277-284$

Shen Y, Li B (2007) Regularized solution to fast GPS ambiguity resolution. J Surv Eng 133(4):168-172

Teunissen P (1993) Least squares estimation of integer GPS ambiguities. Invited lecture, Sect. IV Theory and Methodology, IAG General Meeting, Beijing

Teunissen P (1995) The least-squares ambiguity decorrelation adjustment: a method for fast GPS integer ambiguity estimation. J Geod 70:65-82

Teunissen P (1997) A canonical theory for short GPS baselines, Part I: The baseline precision. J Geod 71:320-336

Teunissen P (1998) Success probability of integer GPS ambiguity rounding and bootstrapping. J Geod 72:606-612 
Teunissen P (1999) An optimality property of the integer least-squares estimator. J Geod 73:587-593

Teunissen P (2001) Integer estimation in the presence of biases. J Geod 75:399-408

Teunissen P (2002) The parameter distributions of the integer GPS model. J Geod 76:41-48

Teunissen P, Kleusberg A (eds) (1998) GPS for geodesy, 2nd edn. Springer, Heidelberg

Teunissen P, Simons D, Tiberius C (2008) Probability and observation theory. Lecture Notes Delft University of Technology, Delft, The Netherlands.

Tikhonov A (1963) Regularization of ill-posed problems. English translation of Dokl. Akad. Nauk. SSSR, 151(1):49-52

Verhagen S (2004) Integer ambiguity validation: an open problem? GPS Solut 8(1):36-43

Verhagen S (2005) The GNSS integer ambiguities: estimation and validation, PhD thesis, Delft University of Technology

Weinziel S (2000) Introduction to Monte Carlo methods, 23 Jun 2000. http://arxiv.org/abs/hep-ph/0006269.

Wübbena G (1989) The GPS adjustment software package-GEONAP concepts and models. Proc 5th Geod Symp Satellite Positioning 452-461

Xu P (1998a) Mixed integer geodetic observation models and integer programming with applications to GPS ambiguity resolution. J Geod Soc Jaoan, 44(3):169-187

Xu P (1998b) Truncated SVD methods for discrete linear ill-posed problems. Geophys J Int 135:505-514

Xu P (2001) Random simulation and GPS decorrelation. J Geod 75:408-423

Xu P (2006) Voronoi cells, probabilistic bounds and hypothesis testing in mixed integer linear models. IEEE Trans on Infor Theory 52(2):3122-3138

Xu P, Cannon E, Lachapelle G (1995) Mixed integer programming for the resolution of GPS carrier phase ambiguities. Presented at the IUGG95 Assembly, Boulder, 2-14 July

Xu P, Cannon E, Lachapelle G (1999) Stabilizing ill-conditioned linear complementarity problems. J Geod 73(4):204-213

Xu P, Shen Y, Fukuda Y, Liu Y (2006) Variance component estimation in linear inverse ill-posed models. 80:69-81

Xu P, Rummel R (1994) A simulation study of smoothness methods in recovery of regional gravity fields. Geophys J Int 117:472-486

Zhu L, Lai Y, Shah M, Mahmood S (2007) Efficiency of carrier-phase integer ambiguity resolution for precise GPS positioning in noisy environments. J Geod 81:149-156 